\title{
COUPLING IN THE SINGULAR LIMIT OF THIN QUANTUM WAVEGUIDES
}

\author{
SERGIO ALBEVERIO, CLAUDIO CACCIAPUOTI, AND DOMENICO FINCO
}

\begin{abstract}
We analyze the problem of approximating a smooth quantum waveguide with a quantum graph. We consider a planar curve with compactly supported curvature and a strip of constant width around the curve. We rescale the curvature and the width in such a way that the strip can be approximated by a singular limit curve, consisting of one vertex and two infinite, straight edges, i.e. a broken line. We discuss the convergence of the Laplacian, with Dirichlet boundary conditions on the strip, in a suitable sense and we obtain two possible limits: the Laplacian on the line with Dirichlet boundary conditions in the origin and a non trivial family of point perturbations of the Laplacian on the line. The first case generically occurs and corresponds to the decoupling of the two components of the limit curve, while in the second case a coupling takes place. We present also two families of curves which give rise to coupling.
\end{abstract}

\section{INTRODUCTION}

In many microscopic systems a quantum particle is constrained by a confining potential to a region with transversal dimensions small with respect to the longitudinal ones. For example in organic molecules the atoms make strong bonds and organize themselves on a regular structure, then the $\pi$-electrons move in correspondence of the bonds under the action of a strong confining potential. Since the early 50s one dimensional models were used to describe the dynamics of $\pi$-electrons in such molecules (see, e.g., [26]).

In more recent times a growing interest in the quantum dynamics of particles in quasi one dimensional structures has been driven by the possibility to realize devices with transversal dimensions on the scale of length of hundreds of nanometers, such as nanotubes or quantum wires. The possibility of a "nanotechnology" was already envisaged by R. Feynman in 1959 (see [12]), but the turning point can be fixed in 1981 when G. Binnig and H. Rohrer, of IBM's Zürich Lab, invented the scanning tunneling microscope, making it possible to inspect and manipulate matter on the atomic scale.

Quantum-graphs represent an excellent model for many quasi one dimensional structures like organic molecules, nanotubes and systems of quantum wires. In mathematical terms a quantum-graph is realized by a graph (i.e. a set of points, the vertices, and a set of finite or infinite segments that connect the vertices, the edges), together with a quantum dynamics for a particle on the graph generated by self-adjoint differential or pseudo-differential operators on the graph (see [16, [17] and [18]).

From the point of view of mathematical physics it is an open question to understand in which sense the one dimensional dynamics on a quantum graph approximates the dynamics of a particle constrained on a region with small transversal dimensions. Essentially one can isolate two problems: to determine which one dimensional, differential or pseudo-differential operators are most suitable in order to describe the dynamics on the edges and which couplings in the vertices among the edges can be physically feasible.

2000 Mathematics Subject Classification. 81Q10, 47A10, 35P05.

Key words and phrases. Quantum waveguides, quantum graphs, spectral theory, Kirchhoff rule, Dirichlet boundary conditions, collapsing mesoscopic systems.

The authors thank Pavel Exner and Rodolfo Figari for useful comments and discussions.

This work was supported by the EU-Project "Quantum Probability with Applications to Physics, Information Theory and Biology", by the Collaborative Research Center (SFB) 611 "Singular Phenomena and Scaling in Mathematical Models" and by the Deutsche Forschungsgemeinschaft (DFG). 
A strategy to approach both these open problems, in the case of differential operators, consists in studying the limit of the operator minus the Laplacian defined on two or three dimensional domains with a graph-like topology but finite width, when the width goes to zero.

This paper deals with the problem of the coupling in the vertices. For this reason we want to consider the simplest possible limit dynamics on the edges. We take a planar domain of constant width and which is straight outside a compact region. It is known that for such a kind of domain the limit dynamics on the straight part of the edges will be generated by the one dimensional Laplacian (see e.g. [19, 25, 27, 29, 23] for the Neumann case, 22] for the Dirichlet case with a narrowing producing decoupling, and [7] for the case with quadratic confining potentials).

In the case of a graph with the free Laplacian on the edges there exists a complete characterization of all the possible couplings in the vertices (see [15]). To define the coupling in a vertex there are $n^{2}$ real parameters at disposal, where $n$ is the number of the edges connected with the vertex. It is not clear which boundary conditions can be obtained as the result of taking the zero width limit from a strip or a cylinder around the graph and how the parameters are related to physical properties of the system such as the geometry of the graph (see, e.g., 11] and the appendix by P. Exner in [2]).

The problem of the convergence in the vertices strongly depends on the conditions imposed on the boundary of the domain with finite thickness. Some well established results exist in the case with Neumann boundary conditions (see, e.g., 6], 9], 19], 23], 25], 27]). All the results indicate that the coupling in the vertices is of Kirchhoff type, i.e., the wave function is continuous in the vertices and the sum over all the first derivatives of the wave functions on the edges connected to a vertex is equal to zero.

Analogous results do not exist for the case with Dirichlet boundary conditions. This case is discussed, for vertices with any number of edges, in the work by O. Post [22. There the problem of a manifold shrinking to a graph is analyzed. Under the hypothesis that the manifold narrows around the vertices, it is proved that the spectrum of the operator minus the Laplacian on the manifold converges to the spectrum of minus the Laplacian on the graph with decoupling boundary conditions in the vertices, i.e. wave function equal to zero in the vertices. See also the work by S. Molchanov and B. Vainberg [20] for the analysis of the scattering problem in the Dirichlet case.

The case with Dirichlet boundary conditions is physically very relevant and of great interest, because it correctly describes particles confined in a bounded region. It is reasonable to believe that, also in this case, it is possible to obtain non decoupling conditions in the vertices.

The difficulties arising in the Dirichlet case, with respect to the Neumann one, are related to the spectrum of the Laplacian on compact domains. Only in the Neumann case, zero is an eigenvalue and the constant function is the corresponding eigenfunction. The occurrence of the eigenvalue zero makes it possible to approximate the wave function by a constant in a small neighborhood of the vertices, that is crucial to prove the convergence to Kirchhoff type conditions in the vertices. Such a simple approximation does not hold in the Dirichlet case and at the present time a reasonable guess on how to approximate the wave function near the vertices is lacking. As a first step in the analysis of the Dirichlet case we consider a simple case of a planar quantum waveguide, i.e. we consider a strip in the plane with constant width around a smooth curve and we take the Laplacian with Dirichlet boundary conditions on this domain. In such a case it is possible to define a system of global coordinates given by the arc length of the curve and the distance from the curve (such a natural system of global coordinates does not exist for a general domain).

In our model the quantum waveguide will "collapse" on a "prototypical" quantum graph made up of a broken line, this is achieved with a suitable scaling of the width and of the curvature of the strip. We assume that the curve is a straight line outside of a compact region, i.e. the signed curvature $\gamma(t), t \in \mathbb{R}$, is a function with compact support. We introduce a dimensionless scaling parameter, $\varepsilon$, and assume that the width of the waveguide scales as $\varepsilon^{\alpha} d$, where $d$ is a positive constant and $\alpha \geqslant 1$, while the curvature scales as $\varepsilon^{-1} \gamma(t / \varepsilon)$. Under these assumptions, when $\varepsilon$ goes to zero, the waveguide narrows to a one dimensional domain made up 
of two straight lines with the same origin. Let us notice that with this scaling the angle between the straight parts of the curve is fixed.

Our main result, stated in theorem 1, is the following: for $\alpha>5 / 2$ generically the limit operator corresponds to the free Laplacian with decoupling boundary conditions in the origin; nevertheless, if the curvature is such that the one dimensional Hamiltonian $-\Delta-\gamma^{2} / 4$ has a zero energy resonance, the limit operator is a point perturbation of the Laplacian in dimension one and the boundary conditions are non decoupling.

We prove the uniform convergence of the resolvent. A renormalization of the spectral parameter is necessary because of the divergence of the term corresponding to the kinetic energy associated with the motion in the transversal direction relative to the curve. This renormalization procedure has been used before when dealing with Dirichlet boundary conditions or with quadratic confining potentials (see [7] and [22]).

We consider two examples of curves that generate a non decoupling dynamics. Such examples indicate that the angle $\theta$ between the straight parts of the curve, is not enough to characterize the limit dynamics. An interesting open question is to understand which geometrical quantities are sufficient to characterize the limit dynamics.

For a simple case of a quantum graph we obtained, for the first time, non decoupling boundary conditions in the vertex in the Dirichlet case. The uniform convergence of the resolvent can be a first step to prove the convergence of the dynamics.

Our result is consistent with the one obtained by O. Post (see [22]). In fact in that work the decoupling in the vertices was due to the narrowing of the domain in a neighborhood of the vertices.

Our model is basically the same as in [7. In their paper G. Dell'Antonio and L. Tenuta studied the case in which the particle is confined around a curve by a quadratic potential and they focused their attention on the convergence of the dynamics on the strip to the dynamics on the broken line. As intermediate step they proved that the quadratic confining potential is equivalent to a domain with Dirichlet boundary condition. Moreover they proved that in the general case the limit dynamics is decoupling.

It is not trivial to extend our result to the case of three or more edges connected to the same vertex, a result weaker than the convergence of the resolvent (e.g. the convergence of the spectrum) would already be of great interest. Nevertheless we think that, similarly as in the case we discuss here, the role played by the resonances will be decisive, even for proving a weaker result.

The paper is organized as follows. In section 2 we describe in detail our model and we state the main theorem. The section 3 is devoted to the proof of the main theorem. In section 4 we give a characterization of the limit operator: we discuss its spectrum, we give the integral kernel of the propagator and evaluate the elements of the scattering matrix. In the last section we investigate the relation between the curvature and the limit dynamics and describe two examples of curves that generate a non decoupling limit dynamics.

\section{MAIN THEOREM}

In this section we shall present our main theorem. First we shall introduce our model of a quantum strip and we shall recall some basic facts about the low energy expansion of the resolvent of a one dimensional Schrödinger operator.

Let $\Gamma$ be a curve in $\mathbb{R}^{2}$ given in parametric form by $\Gamma=\left\{\left(\gamma_{1}(t), \gamma_{2}(t)\right), t \in \mathbb{R}\right\}$ and let us assume that it is parameterized by the arc length $t$, i.e. $\gamma_{1}^{\prime}(t)^{2}+\gamma_{2}^{\prime}(t)^{2}=1$. We also introduce the signed curvature

$$
\gamma(t)=\gamma_{2}^{\prime}(t) \gamma_{1}^{\prime \prime}(t)-\gamma_{1}^{\prime}(t) \gamma_{2}^{\prime \prime}(t)
$$

the curvature radius of $\Gamma$ in $t$ is equal to the inverse of the modulus of the signed curvature.

We shall assume that $\gamma$ has compact support, therefore $\Gamma$ is a straight line outside a compact region. We shall assume also that $\Gamma$ has no self-intersection. Thus $\Gamma$ consists of two straight lines, $l_{1}$ and $l_{2}$, with the origins, $O_{1}$ and $O_{2}$, connected by a piecewise $C^{4}$, non self-intersecting, curve $C$, running in a compact region. The integral of $\gamma$ gives the angle $\theta$ between $l_{1}$ and $l_{2}$.

Let us denote by $\Omega$ the strip of width $2 d>0$ around $\Gamma$ :

$$
\Omega=\left\{(x, y) \text { s.t. } x=\gamma_{1}(t)-s \gamma_{2}^{\prime}(t), y=\gamma_{2}(t)+s \gamma_{1}^{\prime}(t), t \in \mathbb{R}, s \in[-d, d]\right\} \text {. }
$$


We assume $\sup _{t}|\gamma(t)| d<1$, in this way $(t, s)$ provide a global system of coordinates in $\Omega$.

We denote the Laplacian with Dirichlet boundary condition on $\partial \Omega$ by $-\Delta_{\Omega}^{D} ;-\Delta_{\Omega}^{D}$ is defined as the Friedrichs extension of $-\Delta$ with domain $C_{0}^{\infty}(\Omega \backslash \partial \Omega)$.

It is convenient to write $-\Delta_{\Omega}^{D}$ in terms of the curvilinear coordinates $(t, s)$. In particular the following proposition holds (see [8] and [10 for more details)

Proposition 1. Assume that $\Gamma$ has no self-intersections, let $\gamma$ be piecewise $C^{2}$ with compact support and $\gamma^{\prime}, \gamma^{\prime \prime}$ be bounded, then $-\Delta_{\Omega}^{D}$ is unitarily equivalent to the operator $H$ which is defined as the closure of the essentially self-adjoint operator $H_{0}$ acting on $L^{2}(\mathbb{R} \times[-d, d])$ defined by

with

$$
H_{0}=-\frac{\partial}{\partial t} \frac{1}{(1+s \gamma(t))^{2}} \frac{\partial}{\partial t}-\frac{\partial^{2}}{\partial s^{2}}+V(t, s), \quad t \in \mathbb{R}, s \in[-d, d],
$$

and domain given by

$$
V(t, s)=-\frac{\gamma(t)^{2}}{4(1+s \gamma(t))^{2}}+\frac{s \gamma^{\prime \prime}(t)}{2(1+s \gamma(t))^{3}}-\frac{5}{4} \frac{s^{2} \gamma^{\prime}(t)^{2}}{(1+s \gamma(t))^{4}}
$$

$$
\mathscr{D}\left(H_{0}\right)=\left\{\psi \in L^{2}(\mathbb{R} \times[-d, d]) \text { s.t. } \psi \in C^{\infty}(\mathbb{R} \times[-d, d]), \psi(t, d)=\psi(t,-d)=0, H_{0} \psi \in L^{2}(\mathbb{R} \times[-d, d])\right\} .
$$

With piecewise $C^{2}$ we mean a function which is continuous, with continuous first derivative and eventually with a finite number of discontinuities in the second derivative.

Let us also introduce the normal modes, that is the orthonormal complete system $\left\{\phi_{n}\right\}$ in $L^{2}([-d, d])$ whose elements satisfy the following equation:

$$
\left\{\begin{array}{l}
-\frac{d^{2}}{d s^{2}} \phi_{n}=\lambda_{n} \phi_{n} \\
\phi_{n}(-d)=\phi_{n}(d)=0
\end{array} \quad n=1,2,3, \ldots .\right.
$$

It is straightforward to compute $\phi_{n}$ and $\lambda_{n}$ explicitly

$$
\lambda_{n}=\left(\frac{n \pi}{2 d}\right)^{2} \quad \phi_{n}(s)= \begin{cases}\frac{1}{d^{1 / 2} \cos \left(\frac{n \pi s}{2 d}\right),} & n \text { odd } \\ \frac{1}{d^{1 / 2}} \sin \left(\frac{n \pi s}{2 d}\right), & n \text { even }\end{cases}
$$

We rescale $\gamma$ and $d$ in the following way:

$$
\left\{\begin{array}{l}
\gamma(t) \longrightarrow \frac{1}{\varepsilon} \gamma\left(\frac{t}{\varepsilon}\right) \quad \varepsilon>0, \alpha \geqslant 1 \\
d \quad \longrightarrow \varepsilon^{\alpha} d
\end{array}\right.
$$

In this way we obtain a family of domains $\Omega_{\varepsilon}$ and of operators $-\Delta_{\Omega_{\varepsilon}}^{D}$ such that $\Omega_{\varepsilon}$ approximates, for $\varepsilon \rightarrow 0$, the broken line of angle $\theta$ made up by $l_{1}$ and $l_{2}$ with the same origin, $O_{1} \equiv O_{2} \equiv O$. Notice that the angle $\theta$ is unchanged by the rescaling. We assume $\alpha \geqslant 1$ such that $(t, s)$ are a system of global coordinates also for $\Omega_{\varepsilon}$. Then by proposition 1 for every $\varepsilon>0$, the operator $-\Delta_{\Omega_{\varepsilon}}^{D}$ is unitarily equivalent to $H_{\varepsilon}$ defined as the closure of the essentially self-adjoint operator $H_{0 \varepsilon}$ given by

with

$$
H_{0 \varepsilon}=-\frac{\partial}{\partial t} \frac{1}{\left(1+\varepsilon^{\alpha-1} s \gamma(t / \varepsilon)\right)^{2}} \frac{\partial}{\partial t}-\frac{1}{\varepsilon^{2 \alpha}} \frac{\partial^{2}}{\partial s^{2}}+\frac{1}{\varepsilon^{2}} V_{\varepsilon}(t, s),
$$

and

$$
V_{\varepsilon}(t, s)=-\frac{\gamma(t / \varepsilon)^{2}}{4\left(1+\varepsilon^{\alpha-1} s \gamma(t / \varepsilon)\right)^{2}}+\frac{\varepsilon^{\alpha-1} s \gamma^{\prime \prime}(t / \varepsilon)}{2\left(1+\varepsilon^{\alpha-1} s \gamma(t / \varepsilon)\right)^{3}}-\frac{5}{4} \frac{\varepsilon^{2 \alpha-2} s^{2} \gamma^{\prime}(t / \varepsilon)^{2}}{\left(1+\varepsilon^{\alpha-1} s \gamma(t / \varepsilon)\right)^{4}}
$$

$$
\mathscr{D}\left(H_{0 \varepsilon}\right)=\left\{\psi \in L^{2}\left(\Omega^{\prime}\right) \text { s.t. } \psi \in C^{\infty}\left(\Omega^{\prime}\right), \psi(t, d)=\psi(t,-d)=0, H_{0 \varepsilon} \psi \in L^{2}\left(\Omega^{\prime}\right)\right\}
$$


where we have put $\Omega^{\prime}=\mathbb{R} \times[-d, d]$.

The normal modes $\phi_{n}$ satisfy the equation

$$
\left\{\begin{array}{l}
-\frac{1}{\varepsilon^{2 \alpha}} \frac{d^{2}}{d s^{2}} \phi_{n}=\lambda_{\varepsilon, n} \phi_{n} \quad n=1,2,3, \ldots \\
\phi_{n}(-d)=\phi_{n}(d)=0
\end{array}\right.
$$

With

$$
\lambda_{\varepsilon, n}=\left(\frac{n \pi}{2 \varepsilon^{\alpha} d}\right)^{2} .
$$

Let us recall some facts about the low energy behavior of one dimensional Hamiltonians (we shall use the results of [5]). We consider the Hamiltonian $\bar{H}$ given by:

$$
\bar{H}=\bar{H}_{0}+\bar{V}, \quad \text { with } \bar{H}_{0}=-\frac{d^{2}}{d t^{2}},
$$

where we assume $\int_{\mathbb{R}} \bar{V}(t) d t \neq 0$ and $e^{a|\cdot|} \bar{V} \in L^{1}(\mathbb{R})$ for some $a>0$; all the following results hold under these assumptions on the potential. Let us denote the free resolvent by $G_{k}=\left(\bar{H}_{0}-k^{2}\right)^{-1}$, its integral kernel is given by

$$
G_{k}\left(t, t^{\prime}\right)=\frac{i}{2 k} e^{i k\left|t-t^{\prime}\right|} \quad k^{2} \in \mathbb{C} \backslash \mathbb{R}^{+}, \operatorname{Im} k>0 .
$$

In order to discuss the low energy behavior of the resolvent $\left(\bar{H}-k^{2}\right)^{-1}$ one reduces the problem to the analysis of the properties of the transition operator $T(k)$

$$
T(k)=\left(1+u G_{k} v\right)^{-1} \quad \operatorname{Im} k \geqslant 0, k \neq 0, k^{2} \notin \Sigma_{p}(\bar{H})
$$

where we introduced the following two functions

$$
v(t)=|\bar{V}(t)|^{1 / 2}, \quad u(t)=\operatorname{sgn}[\bar{V}(t)]|\bar{V}(t)|^{1 / 2} .
$$

and $\Sigma_{p}(\bar{H})$ indicates the point spectrum of $\bar{H}$. For this purpose it is necessary to isolate the singularity of the free resolvent. In fact we put

$$
u G_{k} v=\frac{i}{2 k}(v, \cdot) u+M(k)
$$

where $(\cdot, \cdot)$ indicates the standard scalar product in $L^{2}(\mathbb{R})$. Under our assumptions on $\bar{V}$ the operator $M(k)$ admits the following expansion which converges in the Hilbert-Schmidt norm

$$
M(k)=\sum_{n=0}^{\infty}(i k)^{n} m_{n} \quad m_{n}\left(t, t^{\prime}\right)=-1 / 2 u(t) \frac{\left|t-t^{\prime}\right|^{n+1}}{(n+1) !} v\left(t^{\prime}\right), n=0,1, \ldots
$$

Let us notice that, under our assumptions on $\bar{V},(v, u) \neq 0$, then we can define the following two operators

$$
P=\frac{1}{(v, u)}(v, \cdot) u, \quad Q=1-P
$$

such that

$$
T(k)=\left(1+\frac{i(v, u)}{2 k} P+M(k)\right)^{-1} .
$$

We say that $\bar{H}$ has a zero energy resonance if there exist $\psi_{r} \in L^{\infty}(\mathbb{R}), \psi_{r} \notin L^{2}(\mathbb{R})$ such that $\bar{H} \psi_{r}=0$ in distributional sense; this is equivalent to the existence of $\varphi_{0} \in L^{2}(\mathbb{R})$ such that

$$
\varphi_{0}+Q m_{0} Q \varphi_{0}=0 \text {. }
$$


Furthermore, if $\varphi_{0}$ exists, it is unique, up to a trivial multiplicative constant, and we can define two constants $c_{1}$ and $c_{2}$ by

$$
c_{1}=\frac{\left(v, m_{0} \varphi_{0}\right)}{(v, u)}, \quad c_{2}=\frac{1}{2}\left((\cdot) v, \varphi_{0}\right) .
$$

We can choose $\varphi_{0}$ such that $c_{1}$ and $c_{2}$ are real. Furthermore under our assumptions on $\bar{V}$ the constants $c_{1}$ and $c_{2}$ can not be both zero, in such a case $\psi^{r}$ would be in $L^{2}(\mathbb{R})$ then zero would be an eigenvalue for $\bar{H}$ (see Lemma 2.2. in [5]), but this is impossible under our assumptions on the potential, see Theorem 5.2. in [14]. Let $\bar{H}^{r}$ be the following family of self-adjoint operators depending on $c_{1}$ and $c_{2}$

$$
\begin{gathered}
\mathscr{D}\left(\bar{H}^{r}\right)=\left\{f \in H^{2}(\mathbb{R} \backslash 0) \text { s.t. }\left(c_{1}+c_{2}\right) f\left(0^{+}\right)=\left(c_{1}-c_{2}\right) f\left(0^{-}\right),\left(c_{1}-c_{2}\right) f^{\prime}\left(0^{+}\right)=\left(c_{1}+c_{2}\right) f^{\prime}\left(0^{-}\right)\right\} \\
\bar{H}^{r} f=-\frac{d^{2} f}{d t^{2}} \quad t \neq 0 .
\end{gathered}
$$

The Hamiltonian $\bar{H}^{r}$ is a self-adjoint extension of the symmetric operator $-\Delta$ in dimension one defined on $C_{0}^{\infty}(\mathbb{R} \backslash\{0\})$. This kind of point interactions are usually referred to as "scale invariant" (see [13] and references therein). We refer to [1] for a comprehensive characterization of the point perturbations of the Laplacian in dimension one (see also, e.g., [3]).

We denote the one dimensional Laplacian with Dirichlet boundary conditions at the origin by $\bar{H}^{D}$

$$
\begin{gathered}
\mathscr{D}\left(\bar{H}^{D}\right)=\left\{f \in H^{2}(\mathbb{R} \backslash 0) \cap H^{1}(\mathbb{R}) \text { s.t. } f(0)=0\right\} \\
\bar{H}^{D} f=-\frac{d^{2} f}{d t^{2}} \quad t \neq 0 .
\end{gathered}
$$

We want to discuss the convergence of the resolvent of $-\Delta_{\Omega_{\varepsilon}}^{D}$, as $\varepsilon \rightarrow 0$, to a one dimensional operator on the broken line. Since the proposition 1 holds we can reduce the problem to the analysis of the convergence of $H_{\varepsilon}$. The normal modes $\phi_{n}$ diagonalize the transversal part of the kinetic term in $H_{\varepsilon}$, then they provide a useful reference frame for discussing the limit $\varepsilon \rightarrow 0$. For these reason we shall consider the matrix elements of the resolvent of $H_{\varepsilon}$ with respect to $\phi_{n}$ and $\phi_{m}$ and we shall discuss the limit of these operators.

The term $\varepsilon^{-\alpha}$ in the definition of $\lambda_{\varepsilon, n}$, see formula (1), indicates that the transversal part of the kinetic term of $H_{\varepsilon}$ is divergent. In order to obtain a non trivial limit, following a standard procedure (see, e.g., [7] and [22]), we regularize the resolvent of $H_{\varepsilon}$ by subtracting the divergent eigenvalue $\lambda_{\varepsilon, n}$ from the spectral parameter. We shall prove that only the diagonal elements survive as $\varepsilon \rightarrow 0$.

Under our hypothesis the resolvent of $H_{\varepsilon}$ admits the integral kernel $\left(H_{\varepsilon}-k^{2}-\lambda_{\varepsilon, m}\right)^{-1}\left(t, s, t^{\prime}, s^{\prime}\right)$, see Theorem II.37 in [28, and let us introduce the operator $R_{n, m}^{\varepsilon}\left(k^{2}\right): L^{2}(\mathbb{R}) \rightarrow L^{2}(\mathbb{R})$ whose integral kernel is given by

$$
R_{n, m}^{\varepsilon}\left(k^{2}, t, t^{\prime}\right)=\int_{-d}^{d} d s d s^{\prime} \phi_{n}(s)\left(H_{\varepsilon}-k^{2}-\lambda_{\varepsilon, m}\right)^{-1}\left(t, s, t^{\prime}, s^{\prime}\right) \phi_{m}\left(s^{\prime}\right) .
$$

It is straightforward to notice that $R_{n, m}^{\varepsilon}\left(k^{2}\right)$ are bounded operator valued analytic functions of $k^{2}$, for $k^{2} \in \mathbb{C} \backslash \mathbb{R}$ and $\operatorname{Im} k>0$.

Let us fix some notation: for a given open set $E \subset \mathbb{R}^{n}$ and $p \geq 1$ we denote the norm of $L^{p}(E)$ by $\|\cdot\|_{L^{p}(E)}$, when $E$ is omitted it is understood $E=\mathbb{R}$, furthermore we denote the Banach space of bounded operators from $L^{p}(E)$ to $L^{q}(E), p, q \geq 1$, equipped with its natural norm by $\mathscr{B}\left(L^{p}(E), L^{q}(E)\right)$; we also denote the Hilbert-Schmidt norm for operators in $\mathscr{B}\left(L^{2}, L^{2}\right)$ by $\|\cdot\|_{H S}$. We indicate with $c$ a numerical constant whose value may change from line to line. Moreover, we denote by $\mathrm{u}-\lim _{\varepsilon \rightarrow 0}$ the limit in the uniform topology of $\mathscr{B}\left(L^{2}, L^{2}\right)$.

Now we can state our main theorem.

Theorem 1. Assume that $\Gamma$ has no self-intersections and that $\gamma$ is piecewise $C^{2}$, has compact support and $\gamma^{\prime}, \gamma^{\prime \prime}$ are bounded. Moreover take $\alpha>5 / 2$ and put $\bar{V}=-\gamma^{2} / 4$. Then two cases can occur: 
1) There does not exist a zero energy resonance for the Hamiltonian $\bar{H}$. In such a case

$$
\mathrm{u}-\lim _{\varepsilon \rightarrow 0} R_{n, m}^{\varepsilon}\left(k^{2}\right)=\delta_{n, m}\left(\bar{H}^{D}-k^{2}\right)^{-1} \quad k^{2} \in \mathbb{C} \backslash \mathbb{R}, \operatorname{Im} k>0 .
$$

2) There exists a zero energy resonance for the Hamiltonian $\bar{H}$. In such a case

$$
\mathrm{u}-\lim _{\varepsilon \rightarrow 0} R_{n, m}^{\varepsilon}\left(k^{2}\right)=\delta_{n, m}\left(\bar{H}^{r}-k^{2}\right)^{-1} \quad k^{2} \in \mathbb{C} \backslash \mathbb{R}, \operatorname{Im} k>0 .
$$

Here $\delta_{n, m}$ indicates the Kronecker symbol, i.e. $\delta_{n, m}=0$ if $n \neq m$ and $\delta_{n, n}=1$.

We shall prove theorem 1 in the next section.

The assumptions of theorem 1 are not optimal: one could require that $\gamma$ has a suitable decay at infinity, as for instance in [8] and [10, where it is assumed that $\gamma$ belongs to some weighted $L^{p}$, instead of compact support, but we are not interested in the maximal generality.

\section{Proof of Theorem 1}

In this section we shall prove theorem 1 first we shall prove three lemmas and then the proof of theorem 1 will immediately follow.

We are interested in the limit of the following operator for $\varepsilon \rightarrow 0$

$$
\bar{H}_{\varepsilon}=\bar{H}_{0}+\frac{1}{\varepsilon^{2}} \bar{V}(\cdot / \varepsilon)=-\frac{d^{2}}{d t^{2}}+\frac{1}{\varepsilon^{2}} \bar{V}(t / \varepsilon) .
$$

Before stating our result on the convergence of $\bar{H}_{\varepsilon}$, let us introduce some notation and spend few words on the correspondence between our problem and the low energy expansion of the resolvent $\left(\bar{H}-k^{2}\right)^{-1}$.

Let us assume $k^{2} \in \mathbb{C} \backslash \mathbb{R}$ and $\operatorname{Im} k>0$. Define the dilation operator $U_{\varepsilon}$

$$
\left(U_{\varepsilon} f\right)(t)=\frac{1}{\varepsilon^{1 / 2}} f(t / \varepsilon)
$$

the operator $U_{\varepsilon}$ is unitary on $L^{2}(\mathbb{R})$ and, by using the identity $\bar{H}_{\varepsilon}=\varepsilon^{-2} U_{\varepsilon} \bar{H} U_{\varepsilon}^{*}$, one obtains

$$
\left(\bar{H}_{\varepsilon}-k^{2}\right)^{-1}=\varepsilon^{2} U_{\varepsilon}\left(\bar{H}-\varepsilon^{2} k^{2}\right)^{-1} U_{\varepsilon}^{*} .
$$

Here $^{*}$ indicates the adjoint. The resolvent of $\bar{H}$ can be written as

$$
\left(\bar{H}-k^{2}\right)^{-1}=G_{k}-G_{k} v T(k) u G_{k}
$$

where $G_{k}, T(k), u$ and $v$ were defined in (3), (44) and (5) respectively. By using equation (9) in (8) one obtains the following formula for $\left(\bar{H}_{\varepsilon}-k^{2}\right)^{-1}$

$$
\left(\bar{H}_{\varepsilon}-k^{2}\right)^{-1}=G_{k}-\frac{1}{\varepsilon} A_{\varepsilon}(k) T(\varepsilon k) C_{\varepsilon}(k)
$$

where $A_{\varepsilon}(k)$ and $C_{\varepsilon}(k)$ are defined via their integral kernels

$$
\begin{aligned}
& A_{\varepsilon}\left(k ; t, t^{\prime}\right)=G_{k}\left(t-\varepsilon t^{\prime}\right) v\left(t^{\prime}\right) \\
& C_{\varepsilon}\left(k ; t, t^{\prime}\right)=u(t) G_{k}\left(\varepsilon t-t^{\prime}\right) .
\end{aligned}
$$

To obtain the limit of the resolvent (10) we shall use the results of [5] about the low energy behavior of $T(k)$, we recall such results in the following

Proposition 2. Let $\int_{\mathbb{R}} \bar{V}(t) d t \neq 0$ and $e^{a|\cdot|} \bar{V} \in L^{1}(\mathbb{R})$ for some $a>0$. Then two cases can occur:

1) There does not exist a zero energy resonance for the Hamiltonian $\bar{H}$. In such a case no solution, $\varphi_{0} \in L^{2}(\mathbb{R})$, of equation (6) exists. 
2) There exists a zero energy resonance for the Hamiltonian $\bar{H}$. In such a case a solution, $\varphi_{0} \in L^{2}(\mathbb{R})$, of equation (6) exists and the constants $c_{1}$ and $c_{2}$ defined in (7) do not vanish simultaneously.

The operator $T(k)$ has the following norm convergent series expansion around $k=0$

$$
T(k)=\sum_{n=p}^{\infty}(i k)^{n} t_{n}
$$

with $p=0$ in case 1 and $p=-1$ in case 2.

For the proof of proposition 2 we refer to [5]. There the authors give also some recursive formulas to get all the terms $t_{j}$ of the series expansion.

Now we can state and prove the following lemma on the convergence of $\left(\bar{H}_{\varepsilon}-k^{2}\right)^{-1}$ :

Lemma 1. Let $\int_{\mathbb{R}} \bar{V}(t) d t \neq 0$ and $e^{a|\cdot|} \bar{V} \in L^{1}(\mathbb{R})$ for some $a>0$. Then two cases can occur:

1) There does not exist a zero energy resonance for the Hamiltonian $\bar{H}$. In such a case

$$
\mathrm{u}-\lim _{\varepsilon \rightarrow 0}\left(\bar{H}_{\varepsilon}-k^{2}\right)^{-1}=\left(\bar{H}^{D}-k^{2}\right)^{-1} \quad k^{2} \in \mathbb{C} \backslash \mathbb{R}, \operatorname{Im} k>0 .
$$

2) There exists a zero energy resonance for the Hamiltonian $\bar{H}$. In such a case

$$
\mathrm{u}-\lim _{\varepsilon \rightarrow 0}\left(\bar{H}_{\varepsilon}-k^{2}\right)^{-1}=\left(\bar{H}^{r}-k^{2}\right)^{-1} \quad k^{2} \in \mathbb{C} \backslash \mathbb{R}, \operatorname{Im} k>0
$$

Proof. Let us consider first the case 2. Under the assumptions on $\bar{V}(t)$ and for $\operatorname{Im} k>0$ the operators $A_{\varepsilon}(k)$ and $C_{\varepsilon}(k)$ are Hilbert-Schmidt and

$$
\begin{aligned}
& A_{\varepsilon}\left(k ; t, t^{\prime}\right)=\left(\frac{i}{2 k} e^{i k|t|}-\frac{1}{2} e^{i k|t|}\left(\left|t-\varepsilon t^{\prime}\right|-|t|\right)+a_{\varepsilon}\left(k ; t, t^{\prime}\right)\right) v\left(t^{\prime}\right) \\
& C_{\varepsilon}\left(k ; t, t^{\prime}\right)=u(t)\left(\frac{i}{2 k} e^{i k\left|t^{\prime}\right|}-\frac{1}{2} e^{i k\left|t^{\prime}\right|}\left(\left|\varepsilon t-t^{\prime}\right|-\left|t^{\prime}\right|\right)+c_{\varepsilon}\left(k ; t, t^{\prime}\right)\right)
\end{aligned}
$$

where

$$
\begin{aligned}
& a_{\varepsilon}\left(k ; t, t^{\prime}\right)=-\frac{i k}{2} e^{i k|t|} \int_{0}^{\left|t-\varepsilon t^{\prime}\right|-|t|} e^{i k \tau}\left(\left|t-\varepsilon t^{\prime}\right|-|t|-\tau\right) d \tau \\
& c_{\varepsilon}\left(k ; t, t^{\prime}\right)=-\frac{i k}{2} e^{i k\left|t^{\prime}\right|} \int_{0}^{\left|\varepsilon t-t^{\prime}\right|-\left|t^{\prime}\right|} e^{i k \tau}\left(\left|\varepsilon t-t^{\prime}\right|-\left|t^{\prime}\right|-\tau\right) d \tau .
\end{aligned}
$$

The following estimates for $a_{\varepsilon}(k)$ and $c_{\varepsilon}(k)$ hold

$$
\begin{aligned}
& \left\|a_{\varepsilon}(k) v\right\|_{H S} \leqslant \frac{|k|}{4} \frac{1}{\sqrt{\operatorname{Im} k}}\left\|(\cdot)^{2} v\right\|_{L^{2}} \varepsilon^{2} \\
& \left\|u c_{\varepsilon}(k)\right\|_{H S} \leqslant \frac{|k|}{4} \frac{1}{\sqrt{\operatorname{Im} k}}\left\|(\cdot)^{2} u\right\|_{L^{2}} \varepsilon^{2} .
\end{aligned}
$$

From proposition 2 it follows that

$$
T(\varepsilon k)=\frac{1}{i k \varepsilon} t_{-1}+t_{0}+i k \varepsilon t_{1}+b_{\varepsilon}(k)
$$


with $\left\|b_{\varepsilon}(k)\right\|_{\mathscr{B}\left(L^{2}, L^{2}\right)} \leqslant c \varepsilon^{2}$. From (11), (12) and (15) we obtain the following formula for the integral kernel of $A_{\varepsilon}(k) T(\varepsilon k) C_{\varepsilon}(k)$

$$
\begin{aligned}
\left(A_{\varepsilon}(k) T(\varepsilon k) C_{\varepsilon}(k)\right)\left(t, t^{\prime}\right)=\int d \tau d \tau^{\prime}[ & \left(\frac{i}{2 k} e^{i k|t|}-\frac{1}{2} e^{i k|t|}(|t-\varepsilon \tau|-|t|)\right) \times \\
& \times v(\tau)\left(\frac{1}{i k \varepsilon} t_{-1}\left(\tau, \tau^{\prime}\right)+t_{0}\left(\tau, \tau^{\prime}\right)+i k \varepsilon t_{1}\left(\tau, \tau^{\prime}\right)\right) u\left(\tau^{\prime}\right) \times \\
& \left.\times\left(\frac{i}{2 k} e^{i k\left|t^{\prime}\right|}-\frac{1}{2} e^{i k\left|t^{\prime}\right|}\left(\left|\varepsilon \tau^{\prime}-t^{\prime}\right|-\left|t^{\prime}\right|\right)\right)\right]+r_{\varepsilon 1}\left(k ; t, t^{\prime}\right)
\end{aligned}
$$

with $\left\|r_{\varepsilon 1}(k)\right\|_{\mathscr{B}\left(L^{2}, L^{2}\right)} \leqslant c \varepsilon^{2}$. We shall use the following properties of $t_{-1}, t_{0}$ and $t_{1}$

$$
\begin{gathered}
t_{-1} u=0 ; \quad t_{-1}^{*} v=0 ; \quad\left(v, t_{0} u\right)=0 ; \\
\left((\cdot) v, t_{-1} u(\cdot)\right)=\frac{2 c_{2}^{2}}{c_{1}^{2}+c_{2}^{2}} ; \quad\left((\cdot) v, t_{0} u\right)=\left(v, t_{0} u(\cdot)\right)=\frac{2 c_{1} c_{2}}{c_{1}^{2}+c_{2}^{2}} ; \quad\left(v, t_{1} u\right)=-\frac{2 c_{2}^{2}}{c_{1}^{2}+c_{2}^{2}} .
\end{gathered}
$$

For a detailed derivation of (17) and (18) we refer to [5]. Let us state the following equality

$$
|t-\varepsilon \tau|-|t|=-\varepsilon \tau \operatorname{sgn}(t)+2(\varepsilon \tau-t) \mathcal{X}_{[0, \varepsilon \tau]}(t) \Theta(\tau)+2(t-\varepsilon \tau) \mathcal{X}_{[\varepsilon \tau, 0]}(t) \Theta(-\tau)
$$

where $\mathcal{X}_{[a, b]}(t)$ is the characteristic function of the interval $[a, b]$ and $\Theta(\tau)$ is the Heaviside function. The estimates

$$
\begin{aligned}
& \left(\int d t d \tau\left|e^{i k|t|}(\varepsilon \tau-t) \mathcal{X}_{[0, \varepsilon \tau]}(t) \Theta(\tau) v(\tau)\right|^{2}\right)^{1 / 2} \leqslant \varepsilon^{3 / 2}\left\|(\cdot)^{3 / 2} v\right\|_{L^{2}} \\
& \left(\int d t d \tau\left|e^{i k|t|}(t-\varepsilon \tau) \mathcal{X}_{[\varepsilon \tau, 0]}(t) \Theta(-\tau) v(\tau)\right|^{2}\right)^{1 / 2} \leqslant \varepsilon^{3 / 2}\left\|(\cdot)^{3 / 2} v\right\|_{L^{2}} .
\end{aligned}
$$

hold. By using the equality (19) and the estimates (20) and (21), and the corresponding ones for the term $u\left(\tau^{\prime}\right) e^{i k\left|t^{\prime}\right|}\left(\left|\varepsilon \tau^{\prime}-t^{\prime}\right|-\left|t^{\prime}\right|\right)$, in (16) and taking into account equations (17) and (18) we obtain

$$
\begin{aligned}
\left(A_{\varepsilon}(k) T(\varepsilon k) C_{\varepsilon}(k)\right)\left(t, t^{\prime}\right)= & \varepsilon\left(-2 i k \frac{c_{2}^{2}}{c_{1}^{2}+c_{2}^{2}} G_{k}(t) G_{k}\left(t^{\prime}\right)+\frac{2}{i k} \frac{c_{2}^{2}}{c_{1}^{2}+c_{2}^{2}} G_{k}^{\prime}(t) G_{k}^{\prime}\left(t^{\prime}\right)+\right. \\
& \left.-2 \frac{c_{1} c_{2}}{c_{1}^{2}+c_{2}^{2}} G_{k}(t) G_{k}^{\prime}\left(t^{\prime}\right)-2 \frac{c_{1} c_{2}}{c_{1}^{2}+c_{2}^{2}} G_{k}^{\prime}(t) G_{k}\left(t^{\prime}\right)\right)+r_{\varepsilon 2}\left(k ; t, t^{\prime}\right)
\end{aligned}
$$

with $\left\|r_{\varepsilon 2}\right\|_{\mathscr{B}\left(L^{2}, L^{2}\right)} \leqslant c \varepsilon^{3 / 2}$. Here $G_{k}^{\prime}(t)$ is the derivative of $G_{k}(t)$

$$
G_{k}^{\prime}(t)=-\frac{\operatorname{sgn}(t)}{2} e^{i k|t|} \quad k^{2} \in \mathbb{C} \backslash \mathbb{R}^{+}, \operatorname{Im} k>0 .
$$

Then from (10) and (22) it follows that

$$
\mathrm{u}-\lim _{\varepsilon \rightarrow 0}\left(\bar{H}_{\varepsilon}-k^{2}\right)^{-1}=\bar{R}^{r}\left(k^{2}\right) \quad k^{2} \in \mathbb{C} \backslash \mathbb{R}, \operatorname{Im} k>0,
$$

with

$$
\begin{aligned}
\bar{R}^{r}\left(k^{2} ; t, t^{\prime}\right)= & G_{k}\left(t-t^{\prime}\right)+2 i k \frac{c_{2}^{2}}{c_{1}^{2}+c_{2}^{2}} G_{k}(t) G_{k}\left(t^{\prime}\right)-\frac{2}{i k} \frac{c_{2}^{2}}{c_{1}^{2}+c_{2}^{2}} G_{k}^{\prime}(t) G_{k}^{\prime}\left(t^{\prime}\right)+ \\
& +2 \frac{c_{1} c_{2}}{c_{1}^{2}+c_{2}^{2}} G_{k}(t) G_{k}^{\prime}\left(t^{\prime}\right)+2 \frac{c_{1} c_{2}}{c_{1}^{2}+c_{2}^{2}} G_{k}^{\prime}(t) G_{k}\left(t^{\prime}\right) .
\end{aligned}
$$


We need to prove that the operator $\bar{R}^{r}\left(k^{2}\right)$ is the resolvent of the Hamiltonian $\bar{H}^{r}$; in facts we shall prove that $\bar{R}^{r}\left(k^{2}\right)$ is the resolvent of an operator which is a self-adjoint extension of the Laplacian defined on $C_{0}^{\infty}(\mathbb{R} \backslash\{0\})$ and which satisfies the same boundary conditions as $\bar{H}^{r}$ at the origin.

A tedious but straightforward calculation, based on the fact that $G_{k}\left(t-t^{\prime}\right)$ satisfies the resolvent identity, shows that

Moreover

$$
\bar{R}^{r}\left(k^{2}\right)-\bar{R}^{r}\left(p^{2}\right)=\left(k^{2}-p^{2}\right) \bar{R}^{r}\left(p^{2}\right) \bar{R}^{r}\left(k^{2}\right) \quad k^{2}, p^{2} \in \mathbb{C} \backslash \mathbb{R}, \operatorname{Im} k>0, \operatorname{Im} p>0 .
$$

$$
\left(\bar{R}^{r}(z)\right)^{*}=\bar{R}^{r}(\bar{z}) \quad z \in \mathbb{C} \backslash \mathbb{R}, \operatorname{Im} \sqrt{z}>0
$$

where - indicates the complex conjugation. The operator $\bar{R}^{r}\left(k^{2}\right)$ is injective because $G_{k} \notin H^{2}(\mathbb{R})$ and $G_{k}^{\prime} \notin$ $H^{2}(\mathbb{R})$, then it is invertible on $\operatorname{Ran}\left[\bar{R}^{r}\left(k^{2}\right)\right]$ and defines a symmetric operator with domain $\operatorname{Ran}\left[\bar{R}^{r}\left(k^{2}\right)\right]$. Let us define the function $g_{f}(t)=\left(\bar{R}^{r}\left(k^{2}\right) f\right)(t)$, with $f \in L^{2}(\mathbb{R}), k^{2} \in \mathbb{C} \backslash \mathbb{R}, \operatorname{Im} k>0$ :

$$
\begin{aligned}
g_{f}(t)= & \left(G_{k} f\right)(t)+2 i k \frac{c_{2}^{2}}{c_{1}^{2}+c_{2}^{2}} G_{k}(t)\left(G_{k} f\right)(0)+\frac{2}{i k} \frac{c_{2}^{2}}{c_{1}^{2}+c_{2}^{2}} G_{k}^{\prime}(t)\left(G_{k}^{\prime} f\right)(0)+ \\
& -2 \frac{c_{1} c_{2}}{c_{1}^{2}+c_{2}^{2}} G_{k}(t)\left(G_{k}^{\prime} f\right)(0)+2 \frac{c_{1} c_{2}}{c_{1}^{2}+c_{2}^{2}} G_{k}^{\prime}(t)\left(G_{k} f\right)(0)
\end{aligned}
$$

where we used $\int_{\mathbb{R}} G_{k}^{\prime}(\tau) f(\tau) d \tau=-\left(G_{k}^{\prime} f\right)(0)$. A direct computation gives

$$
g_{f}\left(0^{+}\right)=\left(c_{1}-c_{2}\right) K_{f} ; \quad g_{f}\left(0^{-}\right)=\left(c_{1}+c_{2}\right) K_{f} ; \quad g_{f}^{\prime}\left(0^{+}\right)=\left(c_{1}+c_{2}\right) K_{f}^{\prime} ; \quad g_{f}^{\prime}\left(0^{-}\right)=\left(c_{1}-c_{2}\right) K_{f}^{\prime}
$$

with

$$
K_{f}=\left(\frac{c_{1}}{c_{1}^{2}+c_{2}^{2}}\left(G_{k} f\right)(0)-\frac{i}{k} \frac{c_{2}}{c_{1}^{2}+c_{2}^{2}}\left(G_{k}^{\prime} f\right)(0)\right) ; \quad K_{f}^{\prime}=\left(-i k \frac{c_{2}}{c_{1}^{2}+c_{2}^{2}}\left(G_{k} f\right)(0)+\frac{c_{1}}{c_{1}^{2}+c_{2}^{2}}\left(G_{k}^{\prime} f\right)(0)\right) .
$$

Conditions (24) are equivalent to

$$
\left(c_{1}+c_{2}\right) g_{f}\left(0^{+}\right)=\left(c_{1}-c_{2}\right) g_{f}\left(0^{-}\right) ; \quad\left(c_{1}-c_{2}\right) g_{f}^{\prime}\left(0^{+}\right)=\left(c_{1}+c_{2}\right) g_{f}^{\prime}\left(0^{-}\right),
$$

then $\operatorname{Ran}\left[\bar{R}^{r}\left(k^{2}\right)\right]=\mathscr{D}\left(\bar{H}^{r}\right)$. Moreover if $g_{f}(t)=\left(\bar{R}^{r}\left(k^{2}\right) f\right)(t)$ is such that $g_{f}\left(0^{+}\right)=g_{f}\left(0^{-}\right)=g_{f}^{\prime}\left(0^{+}\right)=$ $g_{f}^{\prime}\left(0^{-}\right)=0$, then equations (24) together with the definitions of $K_{f}$ and $K_{f}^{\prime}$ give $\left(G_{k} f\right)(0)=\left(G_{k}^{\prime} f\right)(0)=0$, from which $g_{f}(t)=\left(G_{k} f\right)(t)$. This means that the operator with resolvent $\bar{R}^{r}\left(k^{2}\right)$ acts as the Laplacian on functions with support that does not contain the origin, since its domain coincides with $\mathscr{D}\left(\bar{H}^{r}\right)$ it coincides with $\bar{H}^{r}$.

Alternatively, since $\bar{H}^{r}$ is a self-adjoint extension of the operator $-\Delta$ with $\mathscr{D}(-\Delta)=C_{0}^{\infty}(\mathbb{R} \backslash 0)$, one can prove that $\bar{R}^{r}\left(k^{2}\right)$ is the integral kernel of the resolvent of $H^{r}$ by using the results of [1] or the "modified Krein's resolvent formula" derived in [4] (see also [21]).

The proof of the case 1 is analogous. If there is not a zero energy resonance the series expansion of $T(\varepsilon k)$ starts from the order zero in $\varepsilon$, and the following equations replace the ones in (17) and (18)

$$
\left(v, t_{0} u\right)=0 ; \quad\left((\cdot) v, t_{0} u\right)=\left(v, t_{0} u(\cdot)\right)=0 ; \quad\left(v, t_{1} u\right)=-2 .
$$

Then in such a case the following expansion holds

$$
\left(A_{\varepsilon}(k) T(\varepsilon k) C_{\varepsilon}(k)\right)\left(t, t^{\prime}\right)=-2 i k \varepsilon \frac{i}{2 k} e^{i k|t|} \frac{i}{2 k} e^{i k\left|t^{\prime}\right|}+r_{\varepsilon 3}\left(k ; t, t^{\prime}\right), \quad \text { with }\left\|r_{\varepsilon 3}(k)\right\|_{\mathscr{B}\left(L^{2}, L^{2}\right)} \leqslant c \varepsilon^{3 / 2}
$$

and

where

$$
\mathrm{u}-\lim _{\varepsilon \rightarrow 0}\left(\bar{H}_{\varepsilon}-k^{2}\right)^{-1}=\bar{R}^{D}\left(k^{2}\right) \quad k^{2} \in \mathbb{C} \backslash \mathbb{R}, \operatorname{Im} k>0
$$

$\bar{R}^{D}\left(k^{2}\right)$ is the resolvent of $\bar{H}^{D}$, and the proof of the lemma is complete. 
Let us prove two technical estimates that will be used in lemma 3 .

Lemma 2. Let $\int_{\mathbb{R}} \bar{V}(t) d t \neq 0$ and $e^{a|\cdot|} \bar{V} \in L^{1}(\mathbb{R})$ for some $a>0$ then

$$
\begin{gathered}
\limsup _{\varepsilon \rightarrow 0} \varepsilon^{1 / 2}\left\|\frac{d}{d t}\left(\bar{H}_{\varepsilon}-k^{2}\right)^{-1}\right\|_{\mathscr{B}\left(L^{2}, L^{2}\right)} \leqslant c \\
\limsup _{\varepsilon \rightarrow 0}\left\|\left(\bar{H}_{\varepsilon}-k^{2}\right)^{-1}\right\|_{\mathscr{B}\left(L^{2}, L^{\infty}\right)} \leqslant c
\end{gathered}
$$

Proof. We shall first prove the estimate (26). We use formula (10) and remark that the derivative of the resolvent of the free Laplacian, $G_{k}^{\prime}$, is bounded in $\mathscr{B}\left(L^{2}, L^{2}\right)$. Let us consider the derivative of $A_{\varepsilon}(k) T(\varepsilon k) C_{\varepsilon}(k)$. The case with resonance and the case without resonance must be discussed separately. Let us assume that $\bar{H}$ has a zero energy resonance, by using equations (11), (12) and (15) we obtain

$$
\begin{aligned}
\frac{\partial}{\partial t}\left(A_{\varepsilon}(k) T(\varepsilon k) C_{\varepsilon}(k)\right)\left(t, t^{\prime}\right)=\int d \tau d \tau^{\prime}[ & i k \operatorname{sgn}(t-\varepsilon \tau)\left(\frac{i}{2 k} e^{i k|t|}-\frac{1}{2} e^{i k|t|}(|t-\varepsilon \tau|-|t|)+a_{\varepsilon}(k ; t, \tau)\right) \times \\
& \times v(\tau)\left(\frac{1}{i k \varepsilon} t_{-1}\left(\tau, \tau^{\prime}\right)+t_{0}\left(\tau, \tau^{\prime}\right)+i k \varepsilon t_{1}\left(\tau, \tau^{\prime}\right)+b_{\varepsilon}(k ; t, \tau)\right) u\left(\tau^{\prime}\right) \times \\
& \left.\times\left(\frac{i}{2 k} e^{i k\left|t^{\prime}\right|}-\frac{1}{2} e^{i k\left|t^{\prime}\right|}\left(\left|\varepsilon \tau^{\prime}-t^{\prime}\right|-\left|t^{\prime}\right|\right)+c_{\varepsilon}(k ; t, \tau)\right)\right] .
\end{aligned}
$$

Following what was done in the lemma 1 we use the identity (19) and the properties of operators $t_{-1}, t_{0}$ and $t_{1}$, see (17) and (18), to obtain

$$
\begin{aligned}
\frac{\partial}{\partial t}\left(A_{\varepsilon}(k) T(\varepsilon k) C_{\varepsilon}(k)\right)\left(t, t^{\prime}\right)= & \frac{i}{4 k} e^{i k|t|} \int \operatorname{sgn}(t-\varepsilon \tau) v(\tau) t_{-1}\left(\tau, \tau^{\prime}\right) u\left(\tau^{\prime}\right) \tau^{\prime} d \tau d \tau^{\prime} \operatorname{sgn}\left(t^{\prime}\right) e^{i k\left|t^{\prime}\right|}+ \\
& -\frac{i}{4 k} e^{i k|t|} \int \operatorname{sgn}(t-\varepsilon \tau) v(\tau) t_{0}\left(\tau, \tau^{\prime}\right) u\left(\tau^{\prime}\right) d \tau d \tau^{\prime} e^{i k\left|t^{\prime}\right|}+r_{\varepsilon 4}\left(k ; t, t^{\prime}\right)
\end{aligned}
$$

where, for $\varepsilon$ small enough, $\left\|r_{\varepsilon 4}(k)\right\|_{\mathscr{B}\left(L^{2}, L^{2}\right)} \leqslant c \varepsilon$. Now we use the following expression for the function $\operatorname{sgn}(t-\varepsilon \tau)$

$$
\operatorname{sgn}(t-\varepsilon \tau)=\operatorname{sgn}(t)-2 \mathcal{X}_{[0, \varepsilon \tau]}(t) \Theta(\tau)+2 \mathcal{X}_{[\varepsilon \tau, 0]}(-\tau) .
$$

Since $t_{-1}^{*} v=0,\left(v, t_{0} u\right)=0$ and

$$
\begin{aligned}
& \left(\int d t d \tau\left|e^{i k|t|} \mathcal{X}_{[0, \varepsilon \tau]}(t) \Theta(\tau) v(\tau)\right|^{2}\right)^{1 / 2} \leqslant \varepsilon^{1 / 2}\left\|(\cdot)^{1 / 2} v\right\|_{L^{2}} \\
& \left(\int d t d \tau\left|e^{i k|t|} \mathcal{X}_{[\varepsilon \tau, 0]}(t) \Theta(-\tau) v(\tau)\right|^{2}\right)^{1 / 2} \leqslant \varepsilon^{1 / 2}\left\|(\cdot)^{1 / 2} v\right\|_{L^{2}},
\end{aligned}
$$

for $\varepsilon$ small enough the estimate

$$
\left\|\frac{d}{d t} A_{\varepsilon}(k) T(\varepsilon k) C_{\varepsilon}(k)\right\|_{\mathscr{B}\left(L^{2}, L^{2}\right)} \leqslant c \varepsilon^{1 / 2}
$$

holds, from which the limit estimate (26) immediately follows. The case without resonance is analogous. The only difference is in the series expansion of $T(\varepsilon k)$, in fact the series starts with the term of order zero in $\varepsilon$ and equations (17) and (18) are replaced by (25).

Let us prove the estimate (27). We use again the resolvent formula (10). The resolvent of the free Laplacian, $G_{k}$ satisfies

$$
\left\|G_{k}\right\|_{\mathscr{B}\left(L^{2}, L^{\infty}\right)} \leqslant \frac{1}{2|k| \sqrt{\operatorname{Im} k}} .
$$


Let us set $A_{\varepsilon}(k)=A_{1, \varepsilon}(k)+A_{2, \varepsilon}(k)$, with

$$
\begin{aligned}
& A_{1, \varepsilon}\left(k ; t, t^{\prime}\right)=\frac{i}{2 k} e^{i k|t|} v\left(t^{\prime}\right) \\
& A_{2, \varepsilon}\left(k ; t, t^{\prime}\right)=-\frac{1}{2} e^{i k|t|}\left(\left|t-\varepsilon t^{\prime}\right|-|t|\right) v\left(t^{\prime}\right)+a_{\varepsilon}\left(k ; t, t^{\prime}\right) v\left(t^{\prime}\right),
\end{aligned}
$$

where $a_{\varepsilon}\left(k ; t, t^{\prime}\right)$ was given in (13). A direct computation yields

$$
\left\|A_{2, \varepsilon}(k)\right\|_{\mathscr{B}\left(L^{2}, L^{\infty}\right)} \leqslant \frac{1}{2}\|(\cdot) v\|_{L^{2}} \varepsilon+\frac{|k|}{4}\left\|(\cdot)^{2} v\right\|_{L^{2}} \varepsilon^{2}
$$

Moreover the following limit for the $\mathscr{B}\left(L^{2}, L^{2}\right)$-norm of the operator $T(\varepsilon k) C_{\varepsilon}(k)$ holds

$$
\lim _{\varepsilon \rightarrow 0}\left\|T(\varepsilon k) C_{\varepsilon}(k)\right\|_{\mathscr{B}\left(L^{2}, L^{2}\right)} \leqslant c .
$$

In fact, if there does not exist a zero energy resonance the limit (29) is a consequence of the fact that $\lim _{\varepsilon \rightarrow 0}\|T(\varepsilon k)\|_{\mathscr{B}\left(L^{2}, L^{2}\right)} \leqslant c$ and $\lim _{\varepsilon \rightarrow 0}\left\|C_{\varepsilon}(k)\right\|_{\mathscr{B}\left(L^{2}, L^{2}\right)} \leqslant c$. If there is a zero energy resonance we can use (12) and (15). In such a case equation (29) is a consequence of the fact that $t_{-1} u=0$ and ||$\varepsilon t-t^{\prime}|-| t^{\prime}|| \leqslant \varepsilon|t|$. From (28) and (29) we obtain

$$
\limsup _{\varepsilon \rightarrow 0} \frac{1}{\varepsilon}\left\|A_{2, \varepsilon}(k) T(\varepsilon k) C_{\varepsilon}(k)\right\|_{\mathscr{B}\left(L^{2}, L^{\infty}\right)} \leqslant c .
$$

The limit of $\varepsilon^{-1}\left\|A_{1, \varepsilon}(k) T(\varepsilon k) C_{\varepsilon}(k)\right\|_{\mathscr{B}\left(L^{2}, L^{\infty}\right)}$, as $\varepsilon \rightarrow 0$, can be studied as follows. In the presence of a zero energy resonance the integral kernel of $A_{1, \varepsilon}(k) T(\varepsilon k) C_{\varepsilon}(k)$ is

$$
\begin{aligned}
\left(A_{1, \varepsilon}(k) T(\varepsilon k) C_{\varepsilon}(k)\right)\left(t, t^{\prime}\right)=\frac{i}{2 k} e^{i k|t|} \int d \tau d \tau^{\prime} & {\left[v(\tau)\left(\frac{1}{i k \varepsilon} t_{-1}\left(\tau, \tau^{\prime}\right)+t_{0}\left(\tau, \tau^{\prime}\right)+i k \varepsilon t_{1}\left(\tau, \tau^{\prime}\right)+b_{\varepsilon}\left(k ; \tau, \tau^{\prime}\right)\right) u\left(\tau^{\prime}\right) \times\right.} \\
& \left.\times\left(\frac{i}{2 k} e^{i k\left|t^{\prime}\right|}-\frac{1}{2} e^{i k\left|t^{\prime}\right|}\left(\left|\varepsilon \tau^{\prime}-t^{\prime}\right|-\left|t^{\prime}\right|\right)+c_{\varepsilon}\left(k ; \tau^{\prime}, t^{\prime}\right)\right)\right]
\end{aligned}
$$

The modulus of the integral is of order $\varepsilon$. This statement can be proved by reiterating what was done in lemma 1. for this reason we do not give the details of the proof. The term with $\left|\varepsilon \tau^{\prime}-t^{\prime}\right|-\left|t^{\prime}\right|$ can be rewritten by using formula (19). Then the properties (17) and (18) of the operators $t_{-1}, t_{0}$ and $t_{1}$ can be used to evaluate the term of order $\varepsilon$. The modulus of the remainder is of order $\varepsilon^{3 / 2}$ because $\left\|c_{\varepsilon}(k)\right\|_{\mathscr{B}\left(L^{2}, L^{2}\right)} \leqslant c \varepsilon^{2},\left\|b_{\varepsilon}(k)\right\|_{\mathscr{B}\left(L^{2}, L^{2}\right)} \leqslant c \varepsilon^{2}$ and because the estimates (20) and (21) hold. Then

$$
\limsup _{\varepsilon \rightarrow 0} \frac{1}{\varepsilon}\left\|A_{1, \varepsilon}(k) T(\varepsilon k) C_{\varepsilon}(k)\right\|_{\mathscr{B}\left(L^{2}, L^{\infty}\right)} \leqslant c,
$$

and the estimate (27) immediately follows. If there does not exist a zero energy resonance, the proof of (30) is analogous, but the series expansion of $T(\varepsilon k)$ starts with the term of order zero in $\varepsilon$ and instead of (17) and (18), equations (25) hold.

Let us introduce the operator $H_{\varepsilon}^{\bar{V}}$ defined as the closure of the essentially self-adjoint operator $H_{0 \varepsilon}^{\bar{V}}$ :

and

$$
H_{0 \varepsilon}^{\bar{V}}=-\frac{\partial^{2}}{\partial t^{2}}-\frac{1}{\varepsilon^{2 \alpha}} \frac{\partial^{2}}{\partial s^{2}}+\frac{1}{\varepsilon^{2}} \bar{V}(t / \varepsilon)
$$

$$
\mathscr{D}\left(H_{0 \varepsilon}^{\bar{V}}\right)=\left\{\psi \in L^{2}\left(\Omega^{\prime}\right) \text { s.t. } \psi \in C^{\infty}\left(\Omega^{\prime}\right), \psi(t, d)=\psi(t,-d)=0, H_{0 \varepsilon}^{\bar{V}} \psi \in L^{2}\left(\Omega^{\prime}\right)\right\} .
$$

Consider the matrix elements $R_{n, m}^{\bar{V}, \varepsilon}$ with respect to the normal modes $\phi_{n}$ and $\phi_{m}$ :

$$
R_{n, m}^{\bar{V}, \varepsilon}\left(k^{2} ; t, t^{\prime}\right)=\int_{-d}^{d} d s d s^{\prime} \phi_{n}(s)\left(H_{\varepsilon}^{\bar{V}}-k^{2}-\lambda_{\varepsilon, m}\right)^{-1}\left(t, s, t^{\prime}, s^{\prime}\right) \phi_{m}\left(s^{\prime}\right)
$$


Notice that $R_{n, m}^{\bar{V}, \varepsilon}\left(k^{2}\right)=\delta_{n, m}\left(\bar{H}_{\varepsilon}-k^{2}\right)^{-1}$ since $H_{\varepsilon}^{\bar{V}}$ is a separable Hamiltonian.

Lemma 3. Assume that $\Gamma$ has no self-intersections, $\gamma$ is piecewise $C^{2}$, has compact support and that $\gamma^{\prime}, \gamma^{\prime \prime}$ are bounded. Moreover take $\alpha>5 / 2$ and put $\bar{V}=-\gamma^{2} / 4$. Then

$$
\mathrm{u}-\lim _{\varepsilon \rightarrow 0}\left(R_{n, m}^{\varepsilon}\left(k^{2}\right)-R_{n, m}^{\bar{V}, \varepsilon}\left(k^{2}\right)\right)=0 \quad k^{2} \in \mathbb{C} \backslash \mathbb{R}, \operatorname{Im} k>0 .
$$

Proof. In order to prove (31) it is sufficient to prove

$$
\left|\left(g,\left(R_{n, m}^{\varepsilon}\left(k^{2}\right)-R_{n, m}^{\bar{V}, \varepsilon}\left(k^{2}\right)\right) f\right)\right| \leqslant c \varepsilon^{\alpha-5 / 2}\|g\|_{L^{2}}\|f\|_{L^{2}}
$$

for any $f, g \in C_{0}^{\infty}$. Using the resolvent identity we have

$$
\begin{aligned}
& \left(H_{\varepsilon}-k^{2}-\lambda_{\varepsilon, m}\right)^{-1}-\left(H_{\varepsilon}^{\bar{V}}-k^{2}-\lambda_{\varepsilon, m}\right)^{-1}= \\
& =\left(H_{\varepsilon}-k^{2}-\lambda_{\varepsilon, m}\right)^{-1}\left[\varepsilon^{\alpha-2} b\left(\frac{\cdot}{\varepsilon}, \cdot\right) \frac{\partial}{\partial t}+\frac{1}{\varepsilon^{2}}\left(V\left(\frac{\cdot}{\varepsilon}, \cdot\right)-\bar{V}\left(\frac{\cdot}{\varepsilon}\right)\right)\right]\left(H_{\varepsilon}^{\bar{V}}-k^{2}-\lambda_{\varepsilon, m}\right)^{-1}
\end{aligned}
$$

with $b(t, s)=-2 s \gamma^{\prime}(t) /\left(1+\varepsilon^{\alpha-1} s \gamma(t)\right)^{3}$. Therefore it is sufficient to estimate $I_{1}$ and $I_{2}$ given by

$$
\begin{gathered}
I_{1}=\left(g \otimes \phi_{n},\left(H_{\varepsilon}-k^{2}-\lambda_{\varepsilon, m}\right)^{-1} \varepsilon^{\alpha-2} b\left(\frac{\dot{\varepsilon}}{\varepsilon}, \cdot\right) \frac{\partial}{\partial t}\left(H_{\varepsilon}^{\bar{V}}-k^{2}-\lambda_{\varepsilon, m}\right)^{-1} f \otimes \phi_{m}\right) \\
I_{2}=\left(g \otimes \phi_{n},\left(H_{\varepsilon}-k^{2}-\lambda_{\varepsilon, m}\right)^{-1} \frac{1}{\varepsilon^{2}}\left(V\left(\frac{\dot{\varepsilon}}{\varepsilon}, \cdot\right)-\bar{V}\left(\frac{\cdot}{\varepsilon}\right)\right)\left(H_{\varepsilon}^{\bar{V}}-k^{2}-\lambda_{\varepsilon, m}\right)^{-1} f \otimes \phi_{m}\right)
\end{gathered}
$$

Since $H_{\varepsilon}$ is a separable Hamiltonian we have:

$$
\left(H_{\varepsilon}^{\bar{V}}-k^{2}-\lambda_{\varepsilon, m}\right)^{-1} f \otimes \phi_{m}=\left(\left(\bar{H}_{\varepsilon}-k^{2}\right)^{-1} f\right) \otimes \phi_{m}
$$

Let us discuss $I_{1}$ : using (26), Cauchy-Schwarz inequality and the estimate

$$
\left\|\left(H_{\varepsilon}-k^{2}-\lambda_{\varepsilon, m}\right)^{-1}\right\|_{\mathscr{B}\left(L^{2}\left(\Omega^{\prime}\right), L^{2}\left(\Omega^{\prime}\right)\right)} \leqslant\left|\operatorname{Im} k^{2}\right|^{-1}
$$

we have

$$
\begin{aligned}
\left|I_{1}\right| & \leqslant \varepsilon^{\alpha-2}\left|\operatorname{Im} k^{2}\right|^{-1}\|g\|_{L^{2}}\left\|b\left(\frac{\cdot}{\varepsilon}, \cdot\right)\right\|_{L^{\infty}\left(\Omega^{\prime}\right)}\left\|\frac{d}{d t}\left(\bar{H}_{\varepsilon}-k^{2}\right)^{-1} f\right\|_{L^{2}} \\
& \leqslant c \varepsilon^{\alpha-5 / 2}\left|\operatorname{Im} k^{2}\right|^{-1}\|g\|_{L^{2}}\|f\|_{L^{2}},
\end{aligned}
$$

where in the second line of (34) we have used the fact that there exists $\varepsilon_{0}$ such that for $0 \leqslant \varepsilon<\varepsilon_{0}$ we have $\|b(\dot{\bar{\varepsilon}}, \cdot)\|_{L^{\infty}\left(\Omega^{\prime}\right)} \leqslant c<+\infty, \gamma$ being bounded.

Let us discuss $I_{2}$; first we notice that

$$
\begin{aligned}
& \frac{1}{\varepsilon^{2}}\left(V\left(\frac{t}{\varepsilon}, s\right)-\bar{V}\left(\frac{t}{\varepsilon}\right)\right)= \\
& =\varepsilon^{\alpha-3}\left(\frac{\gamma(t / \varepsilon)^{2}\left(2 s \gamma(t / \varepsilon)+\varepsilon^{\alpha-1} s^{2} \gamma(t / \varepsilon)^{2}\right)}{4\left(1+\varepsilon^{\alpha-1} s \gamma(t / \varepsilon)\right)^{2}}+\frac{s \gamma^{\prime \prime}(t / \varepsilon)}{2\left(1+\varepsilon^{\alpha-1} s \gamma(t / \varepsilon)\right)^{3}}-\frac{5}{4} \frac{\varepsilon^{\alpha-1} s^{2} \gamma^{\prime}(t / \varepsilon)^{2}}{\left(1+\varepsilon^{\alpha-1} s \gamma(t / \varepsilon)\right)^{4}}\right)
\end{aligned}
$$

Using the Cauchy-Schwarz inequality, (35), (33) and (27) we have

$$
\begin{aligned}
\left|I_{2}\right| & \leqslant\left|\operatorname{Im} k^{2}\right|^{-1}\|g\|_{L^{2}}\left\|\frac{1}{\varepsilon^{2}}\left(V\left(\frac{\dot{a}}{\varepsilon} \cdot\right)-\bar{V}\left(\frac{\dot{\varepsilon}}{\varepsilon}\right)\right)\left(H_{\varepsilon}^{\bar{V}}-k^{2}-\lambda_{\varepsilon, m}\right)^{-1} f \otimes \phi_{m}\right\|_{L^{2}\left(\Omega^{\prime}\right)} \\
& \leqslant\left|\operatorname{Im} k^{2}\right|^{-1}\|g\|_{L^{2}}\left\|\frac{1}{\varepsilon^{2}}\left(V\left(\frac{\dot{z}}{\varepsilon} \cdot\right)-\bar{V}\left(\frac{\cdot}{\varepsilon}\right)\right)\right\|_{L^{2}\left(\Omega^{\prime}\right)}\left\|\left(H_{\varepsilon}^{\bar{V}}-k^{2}-\lambda_{\varepsilon, m}\right)^{-1} f \otimes \phi_{m}\right\|_{L^{\infty}\left(\Omega^{\prime}\right)} \\
& \leqslant c\left|\operatorname{Im} k^{2}\right|^{-1} \varepsilon^{\alpha-5 / 2}\|g\|_{L^{2}}\|f\|_{L^{2}}
\end{aligned}
$$

Estimate (32) follows from (34) and (36). 
Now we can prove theorem 1

Proof of theorem [1. The proof immediately follows from lemma 1 and lemma 3, Lemma 3] states that $R_{n, m}^{\varepsilon}\left(k^{2}\right)$ and $R_{n, m}^{\bar{V}, \varepsilon}\left(k^{2}\right)$ have the same limit, furthermore we have $R_{n, m}^{\bar{V}, \varepsilon}\left(k^{2}\right)=\delta_{n, m}\left(\bar{H}_{\varepsilon}-k^{2}\right)^{-1}$. Since $\gamma$ has compact support, we can apply lemma 11 to prove the uniform convergence of $\left(\bar{H}_{\varepsilon}-k^{2}\right)^{-1}$ to $\left(\bar{H}_{\varepsilon}^{r}-k^{2}\right)^{-1}$ respectively to $\left(\bar{H}_{\varepsilon}^{D}-k^{2}\right)^{-1}$ depending on the presence or not of a zero energy resonance, and theorem 11 is proved.

\section{Properties of the Hamiltonian $\bar{H}^{r}$}

In this section we characterize the Hamiltonian $\bar{H}^{r}$. We analyze the spectrum, we give the explicit expression for the integral kernel of the propagator and evaluate the scattering matrix.

Proposition 3. The operator $\bar{H}^{r}$ has no point spectrum and no singular continuous spectrum. The continuous spectrum is $[0, \infty)$ and there is a zero energy resonance.

Proof. The resolvent (23) has no poles and therefore $\bar{H}^{r}$ has no point spectrum. The essential spectrum is $[0, \infty)$ since $\bar{R}^{r}\left(k^{2}\right)$ is a compact perturbation of the free resolvent; there is no singular continuous spectrum by theorem XIII.20 in [24] and then the continuous spectrum is $[0, \infty)$.

Take two real numbers $a, b$ such that $a\left(c_{1}-c_{2}\right)=b\left(c_{1}+c_{2}\right)$ and consider $\psi^{r}$ given by:

$$
\psi^{r}(t)= \begin{cases}a & t \leqslant 0 \\ b & t>0\end{cases}
$$

It is straightforward to check that $\psi^{r} \in L^{\infty}$ and that it is a distributional solution of $\bar{H}^{r} \psi^{r}=0$. In fact take

$$
\begin{aligned}
& \mathscr{E}=\left\{\eta \in \mathscr{D}\left(\bar{H}^{r}\right) \text { s.t. } \eta \in C_{0}^{\infty}((-\infty, 0]) \cap C_{0}^{\infty}([0, \infty)),\left(c_{1}+c_{2}\right) \eta\left(0^{+}\right)=\left(c_{1}-c_{2}\right) \eta\left(0^{-}\right),\right. \\
& \left.\left(c_{1}-c_{2}\right) \eta^{\prime}\left(0^{+}\right)=\left(c_{1}+c_{2}\right) \eta^{\prime}\left(0^{-}\right)\right\} \text {. }
\end{aligned}
$$

The set $\mathscr{E}$ is a core for $\bar{H}^{r}$ and integrating by parts we have:

$$
\left(\eta, \bar{H}^{r} \psi^{r}\right)-\left(\bar{H}^{r} \eta, \psi^{r}\right)=a \eta^{\prime}\left(0^{-}\right)-b \eta\left(0^{+}\right)=0 .
$$

for any $\eta \in \mathscr{E}$.

The integral kernel of the one parameter unitary group $e^{-i t \bar{H}^{r}}$ can be derived by using the results of [1] and we obtain:

$$
e^{-i t \bar{H}^{r}}(x, y)=e^{-i t \bar{H}_{0}}(x-y)-\left[\frac{c_{2}^{2}}{c_{1}^{2}+c_{2}^{2}}+\frac{c_{1} c_{2}}{c_{1}^{2}+c_{2}^{2}} \operatorname{sgn} x+\frac{c_{1} c_{2}}{c_{1}^{2}+c_{2}^{2}} \operatorname{sgn} y-\frac{c_{2}^{2}}{c_{1}^{2}+c_{2}^{2}} \operatorname{sgn} x y\right] e^{-i t \bar{H}_{0}}(|x|+|y|)
$$

where $e^{-i t \bar{H}_{0}}(x)=(4 \pi i t)^{-1 / 2} e^{-i \frac{|x|^{2}}{4 t}}$ is the well know propagator of the free Schrödinger equation. It is possible to compute the generalized eigenfunctions: let $p$ a positive number and let us define two family of functions.

$$
\psi_{p}^{+}(x)=\left\{\begin{array}{cc}
e^{i p x}+\frac{2 c_{1} c_{2}}{c_{1}^{2}+c_{2}^{2}} e^{-i p x} & x<0 \\
\frac{c_{1}^{2}-c_{2}^{2}}{c_{1}^{2}+c_{2}^{2}} e^{i p x} & x>0
\end{array}\right.
$$




$$
\psi_{p}^{-}(x)=\left\{\begin{array}{cc}
\frac{c_{1}^{2}-c_{2}^{2}}{c_{1}^{2}+c_{2}^{2}} e^{-i p x} & x<0 \\
e^{-i p x}-\frac{2 c_{1} c_{2}}{c_{1}^{2}+c_{2}^{2}} e^{i p x} & x>0
\end{array}\right.
$$

It is straightforward to check that $\psi_{p}^{+}$and $\psi_{p}^{-}$are linearly independent and satisfy

$$
\bar{H}^{r} \psi_{p}^{ \pm}=p^{2} \psi_{p}^{ \pm}
$$

in a weak sense, as defined in (37). It is immediate to compute the reflection and transmission coefficients $\mathcal{R}_{ \pm}$ and $\mathcal{T}_{ \pm}$from (39) and (38) and we have:

$$
\mathcal{T}_{ \pm}=\frac{c_{1}^{2}-c_{2}^{2}}{c_{1}^{2}+c_{2}^{2}} \quad \mathcal{R}_{ \pm}= \pm \frac{2 c_{1} c_{2}}{c_{1}^{2}+c_{2}^{2}}
$$

Notice that $\mathcal{R}_{ \pm}$and $\mathcal{T}_{ \pm}$do not depend on the energy parameter $p$. The scattering matrix $\mathcal{S}$ is given by:

$$
\mathcal{S}=\left[\begin{array}{cc}
\frac{c_{1}^{2}-c_{2}^{2}}{c_{1}^{2}+c_{2}^{2}} & -\frac{2 c_{1} c_{2}}{c_{1}^{2}+c_{2}^{2}} \\
\frac{2 c_{1} c_{2}}{c_{1}^{2}+c_{2}^{2}} & \frac{c_{1}^{2}-c_{2}^{2}}{c_{1}^{2}+c_{2}^{2}}
\end{array}\right]
$$

\section{EXAMPLES}

In this section we shall present two simple examples and we shall make some remarks about the dependence of the limit operator on the initial curve $\Gamma$.

Let us discuss some properties of symmetric potentials. Assume that $\bar{V}(t)$ satisfies the hypothesis of proposition 2. Assume moreover that it is such that the Hamiltonian $\bar{H}$ defined in (2) has a zero energy resonance and that $\bar{V}(t)=\bar{V}(-t)$. Let us indicate with $\psi^{r}(t)$ the resonance of $\bar{H}$. Since $\bar{V}(t)$ is symmetric the function $\psi^{r}(t)$ has a definite parity. Given $\psi^{r}(t)$, the function $\varphi_{0}(t)$ solution of equation (6) , satisfies $\varphi_{0}(t)=-u(t) \psi^{r}(t)$ a.e. (see Lemma 2.2. in [5]). Since $u(t)$ is symmetric, $\varphi_{0}(t)$ has the same parity as $\psi^{r}(t)$. Then a simple calculation shows that only two boundary conditions for the functions in the domain of the limit operator $\bar{H}^{r}$ are possible

$$
\begin{aligned}
& f\left(0^{-}\right)=f\left(0^{+}\right), \quad f^{\prime}\left(0^{-}\right)=f^{\prime}\left(0^{+}\right), \quad \psi^{r}(t) \text { even } \\
& f\left(0^{-}\right)=-f\left(0^{+}\right), \quad f^{\prime}\left(0^{-}\right)=-f^{\prime}\left(0^{+}\right), \quad \psi^{r}(t) \text { odd } .
\end{aligned}
$$

Let us notice that if $\psi^{r}$ is even the limit operator $\bar{H}^{r}$ is the free Laplacian.

Since $\bar{V}=-\gamma^{2} / 4$ it is clear that the potential does not determine the curvature uniquely. Then we expect that different curves give the same limit operator.

In the following examples we will consider curves for which the curvature is piecewise constant. Before discussing the examples, let us show that, by relaxing the assumption on $\alpha$, it is possible to take into account curves with piecewise constant curvature.

Let us consider the curvature

$$
\gamma(t)= \begin{cases}0 & t \notin[a, b] \\ c_{i} & t \in\left[x_{i-1}, x_{i}\right] \quad i=1, \ldots, n\end{cases}
$$

where $c_{i} \in \mathbb{R}$, and we fix $x_{0}=a$ and $x_{n}=b$. We assume that the constants $c_{i}$ are chosen in such a way that the corresponding curve $\Gamma$ has not self-intersections. The curve corresponding to the curvature (40) is straight outside $[a, b]$ and in every interval $\left[x_{i-1}, x_{i}\right]$ it is the arc of a circumference with radius $\left|c_{i}\right|$. Consider $\beta>0$ and 
$0<\varepsilon<\varepsilon_{0}$ such that $\varepsilon_{0}^{\beta}<1 / 2 \min _{i \neq j}\left|x_{i}-x_{j}\right|$. Let us define the smoothed version of $\gamma(t)$ :

$$
\tilde{\gamma}_{\varepsilon}(t)= \begin{cases}0 & t \notin\left[a-\varepsilon^{\beta}, b+\varepsilon^{\beta}\right] \\ c_{i} & t \in\left[x_{i-1}+\varepsilon^{\beta}, x_{i}-\varepsilon^{\beta}\right] \quad i=1, \ldots, n \\ p_{\varepsilon, i}(t) & t \in\left[x_{i}-\varepsilon^{\beta}, x_{i}+\varepsilon^{\beta}\right] \quad i=0,1, \ldots, n\end{cases}
$$

where the functions $p_{\varepsilon, i}(t)$ are piecewise $C^{2}\left(\left[x_{i}-\varepsilon^{\beta}, x_{i}+\varepsilon^{\beta}\right]\right)$ and

$$
p_{\varepsilon, i}\left(x_{i}-\varepsilon^{\beta}\right)=c_{i}, \quad p_{\varepsilon, i}\left(x_{i}+\varepsilon^{\beta}\right)=c_{i+1}, \quad p_{\varepsilon, i}^{\prime}\left(x_{i} \pm \varepsilon^{\beta}\right)=0 ; \quad n=0,1, \ldots, n,
$$

here $c_{0}=c_{n+1}=0$. The curvature $\tilde{\gamma}_{\varepsilon}(t)$ is piecewise $C^{2}$, and $\tilde{\gamma}_{\varepsilon}^{\prime}$ and $\tilde{\gamma}_{\varepsilon}^{\prime \prime}$ are bounded for every $\varepsilon>0$ moreover the curve $\tilde{\Gamma}_{\varepsilon}$, corresponding to the curvature $\tilde{\gamma}_{\varepsilon}$, has no self-intersections. The functions $p_{\varepsilon, i}(t)$ can be chosen, e.g., in the following way

$$
p_{\varepsilon, i}(t)=\left\{\begin{array}{ll}
\frac{c_{i+1}-c_{i}}{2 \varepsilon^{2 \beta}}\left(t-x_{i}\right)^{2}+\frac{c_{i+1}-c_{i}}{\varepsilon^{\beta}}\left(t-x_{i}\right)+\frac{c_{i+1}+c_{i}}{2} & t \in\left[x_{i}-\varepsilon^{\beta}, x_{i}\right] \\
-\frac{c_{i+1}-c_{i}}{2 \varepsilon^{2 \beta}}\left(t-x_{i}\right)^{2}+\frac{c_{i+1}-c_{i}}{\varepsilon^{\beta}}\left(t-x_{i}\right)+\frac{c_{i+1}+c_{i}}{2} & t \in\left[x_{i}, x_{i}+\varepsilon^{\beta}\right]
\end{array} \quad i=0,1, \ldots, n .\right.
$$

Let us indicate with $\tilde{\Omega}_{\varepsilon}$ the strip of width $2 \varepsilon^{\alpha} d$ defined by "fattening" the smoothed curve and by the rescaling $\tilde{\gamma}_{\varepsilon}(t) \rightarrow \varepsilon^{-1} \tilde{\gamma}_{\varepsilon}(t / \varepsilon)$, as it was done in the definition of $\Omega_{\varepsilon}$, notice that $\tilde{\Omega}_{\varepsilon}$ depends on $\varepsilon$ also because of the explicit dependence of $\tilde{\gamma}_{\varepsilon}(t)$ on $\varepsilon$. For every $\varepsilon>0$ the operator $-\Delta_{\tilde{\Omega}_{\varepsilon}}^{D}$ is unitarily equivalent to $\tilde{H}_{\varepsilon}$ defined as the closure of the essentially self-adjoint operator $\tilde{H}_{0 \varepsilon}$

$$
\tilde{H}_{0 \varepsilon}=-\frac{\partial}{\partial t} \frac{1}{\left(1+\varepsilon^{\alpha-1} s \tilde{\gamma}_{\varepsilon}(t / \varepsilon)\right)^{2}} \frac{\partial}{\partial t}-\frac{1}{\varepsilon^{2 \alpha}} \frac{\partial^{2}}{\partial s^{2}}+\frac{1}{\varepsilon^{2}} \tilde{V}_{\varepsilon}(t, s),
$$

with

and

$$
\tilde{V}_{\varepsilon}(t, s)=-\frac{\tilde{\gamma}_{\varepsilon}(t / \varepsilon)^{2}}{4\left(1+\varepsilon^{\alpha-1} s \tilde{\gamma}_{\varepsilon}(t / \varepsilon)\right)^{2}}+\frac{\varepsilon^{\alpha-1} s \tilde{\gamma}_{\varepsilon}^{\prime \prime}(t / \varepsilon)}{2\left(1+\varepsilon^{\alpha-1} s \tilde{\gamma}_{\varepsilon}(t / \varepsilon)\right)^{3}}-\frac{5}{4} \frac{\varepsilon^{2 \alpha-2} s^{2} \tilde{\gamma}_{\varepsilon}^{\prime}(t / \varepsilon)^{2}}{\left(1+\varepsilon^{\alpha-1} s \tilde{\gamma}_{\varepsilon}(t / \varepsilon)\right)^{4}}
$$

$$
\mathscr{D}\left(\tilde{H}_{0 \varepsilon}\right)=\left\{\psi \in L^{2}\left(\Omega^{\prime}\right) \text { s.t. } \psi \in C^{\infty}\left(\Omega^{\prime}\right), \psi(t, d)=\psi(t,-d)=0, \tilde{H}_{0 \varepsilon} \psi \in L^{2}\left(\Omega^{\prime}\right)\right\} .
$$

Let us prove that the result stated in lemma 3 holds if $H_{\varepsilon}$ is replaced by $\tilde{H}_{\varepsilon}$ and $\bar{V}=-\gamma^{2} / 4$ where $\gamma$ is the piecewise constant function given in (40). Let us define

$$
\tilde{R}_{n, m}^{\varepsilon}\left(k^{2}, t, t^{\prime}\right)=\int_{-d}^{d} d s d s^{\prime} \phi_{n}(s)\left(\tilde{H}_{\varepsilon}-k^{2}-\lambda_{\varepsilon, m}\right)^{-1}\left(t, s, t^{\prime}, s^{\prime}\right) \phi_{m}\left(s^{\prime}\right)
$$

and put $\bar{V}=-\gamma^{2} / 4$ with $\gamma$ defined in (40). As it was done in the proof of lemma 3 we need to estimate

$$
\tilde{I}_{1}=\left(g \otimes \phi_{n},\left(\tilde{H}_{\varepsilon}-k^{2}-\lambda_{\varepsilon, m}\right)^{-1} \varepsilon^{\alpha-2} \tilde{b}_{\varepsilon}(\dot{\bar{\varepsilon}}, \cdot) \frac{\partial}{\partial t}\left(H_{\varepsilon}^{\bar{V}}-k^{2}-\lambda_{\varepsilon, m}\right)^{-1} f \otimes \phi_{m}\right)
$$

and

$$
\tilde{I}_{2}=\left(g \otimes \phi_{n},\left(\tilde{H}_{\varepsilon}-k^{2}-\lambda_{\varepsilon, m}\right)^{-1} \frac{1}{\varepsilon^{2}}\left(\tilde{V}_{\varepsilon}\left(\frac{\dot{\varepsilon}}{\varepsilon}, \cdot\right)-\bar{V}\left(\frac{\dot{\varepsilon}}{\varepsilon}\right)\right)\left(H_{\varepsilon}^{\bar{V}}-k^{2}-\lambda_{\varepsilon, m}\right)^{-1} f \otimes \phi_{m}\right)
$$

with $\tilde{b}_{\varepsilon}(t, s)=-2 s \tilde{\gamma}_{\varepsilon}^{\prime}(t) /\left(1+\varepsilon^{\alpha-1} s \tilde{\gamma}_{\varepsilon}(t)\right)^{3}$. The following estimate for $\tilde{I}_{1}$ holds

$$
\left|\tilde{I}_{1}\right| \leqslant c \varepsilon^{\alpha-5 / 2}\left|\operatorname{Im} k^{2}\right|^{-1}\|g\|_{L^{2}}\|f\|_{L^{2}}\left\|\tilde{b}_{\varepsilon}\left(\frac{\dot{\varepsilon}}{\varepsilon}, \cdot\right)\right\|_{L^{\infty}\left(\Omega^{\prime}\right)} .
$$

Since $\left\|\tilde{\gamma}_{\varepsilon}^{\prime}\right\|_{L^{\infty}\left(\Omega^{\prime}\right)} \leqslant c \varepsilon^{-\beta}$

$$
\left|\tilde{I}_{1}\right| \leqslant c \varepsilon^{\alpha-5 / 2-\beta}\left|\operatorname{Im} k^{2}\right|^{-1}\|g\|_{L^{2}} .
$$


Let us discuss the term $\tilde{I}_{2}$. The following estimate

$$
\left|\tilde{I}_{2}\right| \leqslant c\left|\operatorname{Im} k^{2}\right|^{-1}\|g\|_{L^{2}}\left\|\frac{1}{\varepsilon^{2}}\left(\tilde{V}_{\varepsilon}\left(\frac{\dot{q}}{\varepsilon}, \cdot\right)-\bar{V}\left(\frac{\dot{q}}{\varepsilon}\right)\right)\right\|_{L^{2}\left(\Omega^{\prime}\right)}\|f\|_{L^{2}},
$$

where

$$
\begin{aligned}
& \frac{1}{\varepsilon^{2}}\left(\tilde{V}_{\varepsilon}\left(\frac{t}{\varepsilon}, s\right)-\bar{V}\left(\frac{t}{\varepsilon}\right)\right)= \\
= & \varepsilon^{\alpha-3}\left(\frac{\gamma(t / \varepsilon)^{2}\left(2 s \tilde{\gamma}_{\varepsilon}(t / \varepsilon)+\varepsilon^{\alpha-1} s^{2} \tilde{\gamma}_{\varepsilon}(t / \varepsilon)^{2}\right)}{4\left(1+\varepsilon^{\alpha-1} s \tilde{\gamma}_{\varepsilon}(t / \varepsilon)\right)^{2}}+\frac{s \tilde{\gamma}_{\varepsilon}^{\prime \prime}(t / \varepsilon)}{2\left(1+\varepsilon^{\alpha-1} s \tilde{\gamma}_{\varepsilon}(t / \varepsilon)\right)^{3}}-\frac{5}{4} \frac{\varepsilon^{\alpha-1} s^{2} \tilde{\gamma}_{\varepsilon}^{\prime}(t / \varepsilon)^{2}}{\left(1+\varepsilon^{\alpha-1} s \tilde{\gamma}_{\varepsilon}(t / \varepsilon)\right)^{4}}\right)+ \\
& +\varepsilon^{-2}\left(\frac{\gamma(t / \varepsilon)^{2}-\tilde{\gamma}_{\varepsilon}(t / \varepsilon)^{2}}{4\left(1+\varepsilon^{\alpha-1} s \tilde{\gamma}_{\varepsilon}(t / \varepsilon)\right)^{2}}\right),
\end{aligned}
$$

follows directly from formula (36) and from

$$
\left\|\left(H_{\varepsilon}^{\bar{V}}-k^{2}-\lambda_{\varepsilon, m}\right)^{-1} f \otimes \phi_{m}\right\|_{L^{\infty}\left(\Omega^{\prime}\right)} \leqslant c\|f\|_{L^{2}} .
$$

A direct calculation shows that

$$
\left\|\frac{1}{\varepsilon^{2}}\left(\tilde{V}_{\varepsilon}\left(\frac{\dot{q}}{\varepsilon}, \cdot\right)-\bar{V}\left(\frac{\dot{q}}{\varepsilon}\right)\right)\right\|_{L^{2}\left(\Omega^{\prime}\right)} \leqslant c \varepsilon^{\frac{\beta-3}{2}}+c^{\prime} \varepsilon^{(2 \alpha-3 \beta-5) / 2}
$$

where we used $\left\|\tilde{\gamma}_{\varepsilon}^{\prime}\right\|_{L^{\infty}} \leqslant c \varepsilon^{-\beta}$ and $\left\|\tilde{\gamma}_{\varepsilon}^{\prime \prime}\right\|_{L^{\infty}} \leqslant c \varepsilon^{-2 \beta}$. Then for $\alpha>5 / 2+3 \beta / 2$ and $\beta>3$ the following limit holds

$$
\mathrm{u}-\lim _{\varepsilon \rightarrow 0}\left(\tilde{R}_{n, m}^{\varepsilon}\left(k^{2}\right)-R_{n, m}^{\bar{V}, \varepsilon}\left(k^{2}\right)\right)=0 \quad k^{2} \in \mathbb{C} \backslash \mathbb{R}, \operatorname{Im} k>0 .
$$

The result of lemma 1 holds for the piecewise constant potential $\bar{V}=-\gamma^{2} / 4$ with $\gamma$ defined in (40). Then in the following examples we can consider curves with piecewise constant curvature such that the one dimensional Hamiltonian $\bar{H}=\bar{H}_{0}-\gamma^{2} / 4$ has a zero energy resonance, such examples should be read by tacking into account our comment on the smoothing of curves with piecewise constant curvature.

Let us discuss a simple example of a one parameter family of curves generating the same symmetric potential.

Example 1. The single square well, curves with fixed curvature radius.

Let us consider the potential $\bar{V}(t)$ defined in the following way:

$$
\bar{V}(t)=\left\{\begin{array}{rl}
0 & t \leqslant 0 \\
-a^{2} & 0<t<b \\
0 & t \geqslant b
\end{array}\right.
$$

where $a$ and $b$ are positive real numbers. It is straightforward to prove that $\bar{H}$ has a zero energy resonance if and only if $a b=n \pi, n=1,2, \ldots$. In particular take $a b=\pi$ and let us consider the one parameter family of curves, $\Gamma_{x}$, with curvature $\gamma_{x}$ defined by:

$$
\gamma_{x}(t)=\left\{\begin{array}{rl}
0 & t \leqslant 0 \\
2 a & 0 \leqslant t<x \\
-2 a & x \leqslant t<b \\
0 & t \geqslant b
\end{array}\right.
$$

with $b / 4<x<3 b / 4$. The restriction on the parameter $x$ avoids self-intersections. All the functions $\gamma_{x}$ give the potential (41) and we have $\theta(x)=\int_{\mathbb{R}} \gamma_{x}(t) d t=2 a(2 x-b)$. Then it straightforward to notice that $\theta$ can assume any value between $-\pi$ and $\pi$. 
This example shows that the angle $\theta$ is not sufficient to characterize the limit Hamiltonian since there are infinitely many different curves with different $\theta$ which have the same limit Hamiltonian $\bar{H}^{r}$.

The previous example suggests that there is an even greater freedom in constructing different curves which gives the same limit operator: in facts for any integer $k>0$ and any partition $\mathscr{P}$ of the interval $(0, b)$ into $k$ sub intervals, we can construct a piecewise constant curvature $\gamma_{\mathscr{P}}$; if the corresponding curve $\Gamma_{\mathscr{P}}$ has no self-intersections, then it satisfies the hypothesis of our theorem. All the $\Gamma_{\mathscr{P}}$ yield the same limit Hamiltonian $\bar{H}^{r}$ since they have the same resonant potential $\bar{V}$ and generically these curves will have different $\theta$.

In the previous example it was crucial that $\Gamma$ had a turning point where $\gamma$ changes sign, otherwise the curve $\Gamma$ would have self-intersections. In the following example we shall consider curves such that $\gamma$ has constant sign. Notice that for this class of curves, the potential $\bar{V}$ uniquely determines the curvature. We shall see that also with this restriction the angle $\theta$ is not sufficient to characterize the limit Hamiltonian $\bar{H}^{r}$.

Example 2. The triple (asymmetric) square well, curves with fixed signum of the curvature.

Let us consider the following potential:

$$
\bar{V}(t)=\left\{\begin{array}{rl}
0 & t \leqslant 0 \\
-a_{1}^{2} & -b_{1}<t<0 \\
-a_{2}^{2} & 0 \leqslant t<b_{2} \\
-a_{3}^{2} & b_{2} \leqslant t<b_{2}+b_{3} \\
0 & t \geqslant b_{2}+b_{3}
\end{array}\right.
$$

where $a_{1}, a_{2}, a_{3}, b_{1}, b_{2}, b_{3}$ are real positive numbers. In this example we consider only curves with fixed signum of the curvature, then we assume that the curvature associated to the potential (43) is

$$
\gamma(t)=\left\{\begin{array}{cl}
0 & t \leqslant 0 \\
2 a_{1} & -b_{1}<t<0 \\
2 a_{2} & 0 \leqslant t<b_{2} \\
2 a_{3} & b_{2} \leqslant t<b_{2}+b_{3} \\
0 & t \geqslant b_{2}+b_{3}
\end{array}\right.
$$

It is straightforward but tedious to prove that $\bar{H}$ has a zero energy resonance if and only if the following equation is satisfied:

$$
\begin{aligned}
& a_{1} a_{3} \sin \left(a_{1} b_{1}\right) \sin \left(a_{2} b_{2}\right) \sin \left(a_{3} b_{3}\right)-a_{2} a_{3} \cos \left(a_{1} b_{1}\right) \cos \left(a_{2} b_{2}\right) \sin \left(a_{3} b_{3}\right) \\
& -a_{2}^{2} \cos \left(a_{1} b_{1}\right) \sin \left(a_{2} b_{2}\right) \cos \left(a_{3} b_{3}\right)-a_{1} a_{2} \sin \left(a_{1} b_{1}\right) \cos \left(a_{2} b_{2}\right) \cos \left(a_{3} b_{3}\right)=0 .
\end{aligned}
$$

Since the curvature has definite positive signum, the assumption that the curve is not self-intersecting is equivalent to the condition

$$
\theta=2\left(a_{1} b_{1}+a_{2} b_{2}+a_{3} b_{3}\right)<\pi .
$$

Then we look for solutions of the equation (44) satisfying the condition (45). As a consequence we have that $0<\cos \left(a_{i} b_{i}\right)<1, i=1,2,3$, in such a case equation (44) is equivalent to:

$$
a_{1} a_{3} \tan \left(a_{1} b_{1}\right) \tan \left(a_{2} b_{2}\right) \tan \left(a_{3} b_{3}\right)-a_{2} a_{3} \tan \left(a_{3} b_{3}\right)-a_{2}^{2} \tan \left(a_{2} b_{2}\right)-a_{1} a_{2} \tan \left(a_{1} b_{1}\right)=0 .
$$

It is straightforward to provide infinitely many solutions of (46). Fix $a_{1} b_{1}=\beta_{1}, a_{2} b_{2}=\beta_{2}$ and $a_{3} b_{3}=\beta_{3}$ such that, $\beta_{1}, \beta_{2}$ and $\beta_{3}$ satisfy the condition (45); this can be done fixing $b_{1}, b_{2}$ and $b_{3}$ leaving $a_{1}, a_{2}$ and $a_{3}$ free. Now equation (46) becomes an equation in $a_{1}, a_{2}$ and $a_{3} \operatorname{since} \tan \left(\beta_{1}\right), \tan \left(\beta_{2}\right)$ and $\tan \left(\beta_{3}\right)$ are fixed positive numbers; for instance we can solve it with respect to $a_{1}$ and we obtain:

$$
a_{1}=\frac{a_{2}^{2} \tan \left(\beta_{2}\right)+a_{2} a_{3} \tan \left(\beta_{3}\right)}{\tan \left(\beta_{1}\right)\left(a_{3} \tan \left(\beta_{2}\right) \tan \left(\beta_{3}\right)-a_{2}\right)} .
$$


Every $a_{2}$ and $a_{3}$ such that $a_{3} \tan \left(\beta_{2}\right) \tan \left(\beta_{3}\right)-a_{2}>0$ provide a solution of (44) with a different potential $\bar{V}$ but the same angle $\theta$.

Therefore we have showed that there are infinitely many different curves with the same angle $\theta$ which give different resonant potentials (42) and therefore different limit Hamiltonian $\bar{H}^{r}$.

It is an interesting open question to find which quantities of the curve $\Gamma$ are sufficient to characterize the limit Hamiltonian $\bar{H}^{r}$.

\section{REFERENCES}

[1] S. Albeverio, Z. Brzeźniak, and L. Da̧browski, Fundamental solutions of the heat and Schrödinger equations with point interactions, J. Funct. Anal. 130 (1995), 220-254.

[2] S. Albeverio, F. Gesztesy, R. Høegh-Krohn, and H. Holden, Solvable models in quantum mechanics: Second edition, AMS Chelsea Publ., 2005, with an Appendix by P. Exner.

[3] S. Albeverio and P. Kurasov, Singular perturbations of differential operators: solvable Schrödinger-type operators, Cambridge University Press, 2000.

[4] S. Albeverio and K. Pankrashkin, A remark on Krein's resolvent formula and boundary conditions, J. Phys. A: Math. Gen. 38 (2005), no. 22, 4859-4864.

[5] D. Bollé, F. Gesztesy, and S. F. J. Wilk, A complete treatment of low-energy scattering in one dimension, J. Operator Theory 13 (1985), no. 1, 3-32.

[6] A. I. Bonciocat, Ph.D. Thesis, Universität Bonn (2006), in preparation.

[7] G. Dell'Antonio and L. Tenuta, Quantum graphs as holonomic constraints, J. Math. Phys. 47 (2006), 072102.

[8] P. Duclos and P. Exner, Curvature-induced bound states in quantum waveguides in two and three dimensions, Rev. Math. Phys. 7 (1995), no. 1, 73-102.

[9] P. Exner and O. Post, Convergence of spectra of graph-like thin manifolds, J. Geom. Phys. 54 (2005), 77-115.

[10] P. Exner and P. Šeba, Bound states in curved quantum waveguides, J. Math. Phys. 30 (1989), no. 11, 2574-2580.

[11] _ Free quantum motion on a branching graph, Rep. Math. Phys. 28 (1989), no. 1, 7-26.

[12] R. P. Feynman, There's plenty of room at the bottom, Talk at the annual meeting of the American Physical Society at the California Institute of Technology (Caltech), December 29th, 1959.

[13] P. Hejčík and T. Cheon, Irregular dynamics in a solvable one-dimensional quantum graph, Phys. Lett. A 356 (2006), $290-293$.

[14] A. Jensen and G. Nenciu, A unified approach to resolvent expansions at thresholds, Rev. Math. Phys. 13 (2001), no. 6, 717-754.

[15] V. Kostrykin and R. Schrader, Kirchhoff's rule for quantum wires, J. Phys. A: Math. Gen. 32 (1999), no. 4, 595-630.

[16] P. Kuchment, Graph models for waves in thin structures, Waves Random Media 12 (2002), no. 4, R1-R24.

[17] _ Quantum graphs. I. Some basic structures, Waves Random Media 14 (2004), no. 1, S107-S128.

[18] _ Quantum graphs. II. Some spectral properties of quantum and combinatorial graphs, J. Phys. A: Math. Gen. 38 (2005), no. 22, 4887-4900.

[19] P. Kuchment and H. Zeng, Convergence of spectra of mesoscopic systems collapsing onto a graph, J. Math. Anal. Appl. 258 (2001), no. 2, 671-700.

[20] S. Molchanov and B. Vainberg, Scattering solutions in a network of thin fibers: small diameter asymptotics, arXiv:math-ph/0609021 (2006).

[21] K. Pankrashkin, Resolvents of self-adjoint extensions with mixed boundary conditions, Rep. Math. Phys. 58 (2006), $207-221$.

[22] O. Post, Branched quantum wave guides with Dirichlet boundary conditions: the decoupling case, J. Phys. A: Math. Gen. 38 (2005), no. 22, 4917-4931.

[23] _ Spectral convergence of quasi-one-dimensional spaces, Ann. Henri Poincaré 7 (2006), 933-973.

[24] M. Reed and B. Simon, Methods of modern mathematical physics, vol. IV: Analysis of Operators, Academic Press, New York-San Francisco-London, 1978.

[25] J. Rubinstein and M. Schatzman, Variational problems on multiply connected thin strips. I. Basic estimates and convergence of the Laplacian spectrum, Arch. Ration. Mech. Anal. 160 (2001), no. 4, 271-308.

[26] K. Ruedenberg and C. W. Scherr, Free-electron network model for conjugated systems. I. Theory, J. Chem. Phys. 21 (1953), no. 9, 1565-1581.

[27] Y. Saitō, Convergence of the Neumann Laplacian on shrinking domains, Analysis (Munich) 21 (2001), no. 2, $171-204$.

[28] B. Simon, Quantum mechanics for Hamiltonians defined as quadratic forms, Princeton University Press, Princeton, 1971. 
Albeverio: Institut Für Angewandte Mathematik

Current address: Wegelerstr. 6, 53115 Bonn, Germany

E-mail address: albeverio@wiener.iam.uni-bonn.de

Cacciapuoti: Institut für Angewandte Mathematik

Current address: Wegelerstr. 6, 53115 Bonn, Germany

E-mail address: caccia@na.infn.it

Finco: Institut für Angewandte Mathematik

Current address: Wegelerstr. 6, 53115 Bonn, Germany

E-mail address: finco@wiener.iam.uni-bonn.de 